\title{
Chronic cerebral hypoperfusion causes decrease of O-GIcNAcylation, hyperphosphorylation of tau and behavioral deficits in mice
}

\author{
Yang Zhao ${ }^{1,2}$, Jin-hua Gu ${ }^{2,3}$, Chun-ling Dai ${ }^{2}$, Qun Liu' ${ }^{1}$, Khalid Iqbal ${ }^{2}$, Fei Liu ${ }^{2}$ and Cheng-Xin Gong ${ }^{2}$ * \\ ${ }^{1}$ Department of Neurology, The First Hospital of Jilin University, Changchun, Jilin, China \\ ${ }^{2}$ Department of Neurochemistry, New York State Institute for Basic Research in Developmental Disabilities, Staten Island, NY, USA \\ ${ }^{3}$ Department of Pharmacology, Medical School, Nantong University, Nantong, Jiangsu, China
}

Edited by:

Xiongwei Zhu, Case Western Reserve

University, USA

Reviewed by:

Xuemin Xu, The University of

Tennessee, USA

Xinglong Wang, Case Western

Reserve University, USA

${ }^{*}$ Correspondence:

Cheng-Xin Gong, Department of Neurochemistry, New York State Institute for Basic Research in

Developmental Disabilities, Staten

Island, NY 10314, USA

e-mail: chengxin.gong@csi.cuny.edu
Chronic cerebral hypoperfusion $(\mathrm{CCH})$ is one of the causes of vascular dementia $(\mathrm{VaD})$ and is also an etiological factor for Alzheimer's disease (AD). However, how $\mathrm{CCH}$ causes cognitive impairment and contributes to Alzheimer's pathology is poorly understood. Here we produced a mouse model of $\mathrm{CCH}$ by unilateral common carotid artery occlusion (UCCAO) and studied the behavioral changes and brain abnormalities in mice 2.5 months after UCCAO. We found that $\mathrm{CCH}$ caused significant short-term memory deficits and mild longterm spatial memory impairment, as well as decreased level of protein O-GlcNAcylation, increased level of tau phosphorylation, dysregulated synaptic proteins and insulin signaling, and selective neurodegeneration in the brain. These findings provide mechanistic insight into the effects of $\mathrm{CCH}$ on memory and cognition and the likely link between $\mathrm{AD}$ and VaD.

Keywords: chronic cerebral hypoperfusion, Alzheimer's disease, cognitive impairment, O-GlcNAcylation, tau phosphorylation, synaptic plasticity markers, brain insulin signaling, neurodegeneration

\section{INTRODUCTION}

Vascular dementia $(\mathrm{VaD})$ is the second most common form of dementia after Alzheimer's disease (AD) in older adults (Battistin and Cagnin, 2010), accounting for $\sim 20 \%$ of all dementia cases worldwide (Dubois and Hébert, 2001). VaD is defined as the loss of cognitive function to a degree that interferes daily living activities caused by problems in blood supply to the brain, which injures brain regions important for memory, cognition, and behavior (Roman, 2002). Chronic cerebral hypoperfusion (CCH) is a major cause of $\mathrm{VaD}$ and can result from disorders that affect cerebral vascular system, including hypertension, diabetes, generalized atherosclerosis, and smoking (Meyer et al., 2000; Valerio Romanini et al., 2013). Individuals with CCH usually have cognitive deficits to various degrees. The important role of $\mathrm{CCH}$ in dementia has already immerged to the front edge of neurology research. Studies in the last decade have suggested that $\mathrm{CCH}$ might promote neurodegeneration through neuronal energy failure, production of reactive oxygen species and proinflammatory cytokines by activated microglial cells that, in turn, damage the neuronal cells, and contribute to white matter lesions (Kitagawa et al., 2005; Farkas et al., 2007; Adibhatla and Hatcher, 2008; Urabe, 2012; Bang et al., 2013).

Cerebral vascular abnormalities are also an important contributing factor for AD (Kalaria et al., 2012; Leszek et al., 2012; Sato and Morishita, 2013; Sudduth etal., 2013). It is estimated that as many as $40 \%$ of $\mathrm{AD}$ patients actually have a mixed dementia of $\mathrm{AD}$ and $\mathrm{VaD}$ (Kammoun et al., 2000). AD is characterized by chronic and progressive neurodegeneration leading to progressive cognitive dysfunction and eventually to death of the patients. $\mathrm{AD}$ in most cases is sporadic and is probably caused by multiple factors. $\mathrm{AD}$ is histopathologically characterized by the presence of both intraneuronal neurofibrillary tangles (NTFs) and extracellular senile plaques together with neurofibrillary degeneration in the brain. NFTs are predominantly comprised of aggregated abnormally hyperphosphorylated tau (Grundke-Iqbal et al., 1986a,b), whereas senile plaques are made of aggregated amyloid- $\beta(A \beta)$ peptides. We have previously found that impaired brain glucose metabolism in AD probably lead to abnormal hyperphosphorylation of tau and neurofibrillary degeneration via down-regulation of tau O-GlcNAcylation, a post-translational modification of proteins with $\beta$-N-acetylglucosamine (GlcNAc; Liu et al., 2004, 2009). Thus, we postulate that $\mathrm{CCH}$ might lead to chronic neurodegeneration and cognitive impairment in $\mathrm{AD}$ and $\mathrm{VaD}$ through reduction of cerebral glucose metabolism and consequently downregulation of O-GlcNAcylation and hyperphosphorylation of tau. The present study was aimed to test this hypothesis in a mouse model of $\mathrm{CCH}$.

A common rodent model of $\mathrm{CCH}$ is produced by unilateral common carotid artery occlusion (UCCAO; Levine, 1960; Rice et al., 1981). A 35-55\% decrease of cerebrocortical perfusion is reported in the ipsilateral hemisphere in mice 28 days after UCCAO surgery (Kitagawa etal., 2005). To investigate the role of $\mathrm{CCH}$ in $\mathrm{AD}$-related brain abnormalities and cognitive impairment, we performed UCCAO in adult mice and then studied general and memory behavior, O-GlcNAcylation, tau phosphorylation, synaptic proteins, hexosamine biosynthetic pathway (HBP) and insulin signaling. We found that $\mathrm{CCH}$ caused anxiety and deficits of motor functions and spatial memory, led to a decrease in O-GlcNAcylation and an increase in tau phosphorylation, dysregulated synaptic proteins, and resulted in alterations of the HBP and insulin signaling pathway in the mouse brains. 


\section{MATERIALS AND METHODS ANTIBODIES AND REAGENTS}

Primary antibodies used in this study are listed in Table $\mathbf{1}$. Peroxidase-conjugated anti-mouse and anti-rabbit IgG were obtained from Jackson ImmunoResearch Laboratories (West Grove, PA, USA). The enhanced chemiluminescence (ECL) kit was from Pierce (Rockford, IL, USA). Avidin-biotin complex (ABC) staining system was from Santa Cruz Biotechnology (Santa Cruz, CA, USA). Other chemicals were from Sigma (St. Louis, MO, USA).

\section{ANIMALS AND UCCAO}

Breeding pairs of mice (B6.129) were originally obtained from the Jackson Laboratory (New Harbor, ME, USA), and the mice were bred at our institutional animal colony. They were housed $(4 \sim 5$ animals per cage) with a 12/12 h light/dark cycle and with ad libitum access to food and water. The housing, breeding, and animal experiments were in accordance with the approved protocol from our Institutional Animal Care and Use Committee and with the PHS Policy on Human Care and Use of Laboratory animals (revised March 15, 2010).

Male mice of 4.5-6 months old were randomly divided into two groups. The $\mathrm{CCH}$ group mice $(n=10)$ were subjected to unilateral (right side) common carotid artery (CCA) occlusion, and the control mice $(n=12)$ underwent the same procedure without vessel occlusion (sham surgery). The UCCAO was performed by permanent vessel double ligation under general anesthesia with $2.5 \%$ avertin. Briefly, a ventral midline incision was made to expose the right CCA. The artery was gently isolated from the carotid sheath and vagus nerve and the right CCA was doubly ligated with 9-0 silk suture (Ethicon ${ }^{\circledR}$ ) just below the bifurcation. The midline incision was then closed carefully, and the animals were transferred back into their cages with free access to water and food. One month after the surgery, the mice were subjected to a battery of behavioral tests which lasted for 6 weeks (Figure 1A). After surgery, the body weight and food intake were monitored weekly. After completion of all behavioral tests, the mice were sacrificed by decapitation 2.5 months after the surgery, and the brains were removed immediately. The cerebral cortex and the hippocampus of the right hemispheres of the sham mice and of both hemispheres of the UCCAO mice were dissected, flash frozen in dry ice, and stored in $-80^{\circ} \mathrm{C}$ for biochemical analyses. Some brains were fixed with $4 \%$ paraformaldehyde in $0.1 \mathrm{M} \mathrm{PBS}$, followed by cryoprotection in $30 \%$ sucrose. Coronal sections $(40 \mu \mathrm{m}$ thick) were cut on a freezing microtome. The sections were stored in glycol anti-freeze solution (ethylene glycol, glycerol, and $0.1 \mathrm{M}$ PBS in a 3:3:4 ratio) at $-20^{\circ} \mathrm{C}$ until immunohistochemical staining.

\section{BEHAVIORAL TESTS}

All behavioral tests were performed between 8:00 a.m. and 5:00 p.m. Before the behavioral tests started, the mice were habituated by being handled by experimenters. Before each test, mice were transported to the test room (temperature was $23 \pm 1{ }^{\circ} \mathrm{C}$ ) located inside of the animal colony to acclimatize for $1 \mathrm{~h}$.

\section{Open field}

Exploratory activity, anxiety and locomotor activity were measured using the open field test. The apparatus was constructed of white painted plywood measured $50 \mathrm{~cm} \times 50 \mathrm{~cm}$ as the square arena and $40 \mathrm{~cm}$ high PVC walls. A central square of $20 \mathrm{~cm} \times 20 \mathrm{~cm}$ was surmounted by an automated camera-based video tracking system (ANY-Maze version 4.5 software; Stoelting Co., Wood Dale, IL, USA). Each mouse was placed individually in the arena and allowed to explore for $15 \mathrm{~min}$, and the overall performance including distance traveled, distance in central area, central area entries and time spent in central area were recorded by the video tracking system. After the 15 min test, mice were returned in their home cages. Between test of each mouse, all urines and defecations were cleared from the field, followed by cleaning with $70 \%$ ethyl alcohol and air drying of the field.

\section{Elevated plus maze}

Elevated plus maze measures anxiety induced by open spaces, as well as height related anxiety (Walf and Frye, 2007). The apparatus was an elevated $(80 \mathrm{~cm}$ high) maze consisting of four arms $(30 \mathrm{~cm} \times 5 \mathrm{~cm}$ each) connected by a common $5 \mathrm{~cm} \times 5 \mathrm{~cm}$ central area making the shape of a plus sign. Two of the arms facing opposite were enclosed by $20 \mathrm{~cm}$-high walls and were thus called closed arm (CA), whereas the other two facing arms were open and were thus called open arm (OA). Each mouse was placed onto the central area facing an $\mathrm{OA}$ and allowed to explore the maze for $8 \mathrm{~min}$. The automatic video tracking system was used for collecting behavioral data including the number of CA entries, $\mathrm{OA}$ entries, and time spent in CA and OA. Between test of each mouse, the maze floor was cleaned as described above for the open field.

\section{Novel object recognition}

Mice have a tendency to interact more with a novel object than with a familiar one. This tendency has been used by behavioral pharmacologists and neuroscientists to study learning and memory. A popular protocol for such research is the object recognition task (Bevins and Besheer, 2006). The test apparatus was the same one as used in the open field test described above. The procedure consisted of three phases: habituation phase, sample phase, and test phase. During habituation phase, each mouse was allowed to explore the field in the absence of objects for $15 \mathrm{~min}$ for four consecutive days in order to make the mice become familiar with the field. On the fifth day during the sample phase, two objects were placed symmetrically onto the arena. The mouse was placed at the mid-point of the wall opposite the sample objects with its body parallel to the side walls and its nose pointing away from the objects, allowed to freely explore for $5 \mathrm{~min}$. Time spent exploring the objects was recorded. During the test phase, one of the two objects used in sample phase was randomly replaced by a novel one, the mouse was reintroduced to the arena for 5 min exploration after a 15 min delay. Another 5 min test session was performed $24 \mathrm{~h}$ later for evaluation of long-term memory retention (Ennaceur and Delacour, 1988). Between each mouse, any feces were cleared, and the arena and objects were cleaned with $70 \%$ ethyl alcohol. The video tracking system was used to collect behavioral performances automatically. Time spent exploring 
Table 1 | Primary antibodies used in this study.

\begin{tabular}{|c|c|c|c|c|}
\hline Antibody & Type & Specificity & Phosphorylation sites & Source/Reference \\
\hline HIF-1 $\beta$ & Mono- & $\mathrm{HIF}-1 \beta$ & & Abcam, Cambridge, MA, USA \\
\hline GLUT1 & Poly- & GLUT1 & & Millipore, Temecula, CA, USA \\
\hline GLUT2 & Poly- & GLUT2 & & Milipore, Billerica, MA, USA \\
\hline GLUT3 & Poly- & GLUT3 & & Santa Cruz Biotech., Santa Cruz, CA, USA \\
\hline OGA & Poly- & OGA & & Crawford et al. (2008) \\
\hline CTD110.6 & Mono- & O-GIcNAc & & Covance, Emeryville, CA, USA \\
\hline $\mathrm{RL} 2$ & Mono- & O-GlcNAc & & Affinity Bioreagents, Golden, CO, USA \\
\hline pS199 & Poly- & P-tau & Ser199 & Invitrogen, Grand Island, NY, USA \\
\hline pT231 & Poly- & P-tau & Thr231 & Invitrogen \\
\hline pS262 & Poly- & P-tau & Ser262 & Invitrogen \\
\hline pS396 & Poly- & P-tau & Ser396 & Invitrogen \\
\hline pS404 & Poly- & P-tau & Ser404 & Invitrogen \\
\hline R134d & Poly- & Tau & & Tatebayashi etal. (1999) \\
\hline Synapsin 1 & Poly- & Synapsin 1 & & Santa Cruz Biotech. \\
\hline Synaptophysin & Mono- & Synaptophysin & & Millipore \\
\hline PSD95 & Mono- & PSD95 & & Cell Signaling Tech, Danvers, MA, USA \\
\hline GFAT2 & Poly- & GFAT2 & & Santa Cruz Biotech. \\
\hline P-PI3K p85 & Poly- & P-PI3K (p85) & Tyr458/Tyr199 & Cell Signaling Technology \\
\hline PDK1 & Poly- & PDK1 & & Cell Signaling Technology \\
\hline PDK1 pS241 & Poly- & P-PDK1 & Ser241 & Cell Signaling Technology \\
\hline AKT & Poly- & AKT & & Cell Signaling Technology \\
\hline AKT pS473 & Poly- & P-AKT & Ser473 & Cell Signaling Technology \\
\hline GSK-3 3 & Poly- & GSK-3 $\beta$ & & Cell Signaling Technology \\
\hline 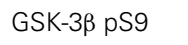 & Poly- & P-GSK-3 $\beta$ & Ser9 & Cell Signaling Technology \\
\hline GAPDH & Poly- & GAPDH & & Santa Cruz Biotech. \\
\hline
\end{tabular}

both the novel and the familiar objects was recorded $\left(T_{N}, T_{F}\right)$. Object discrimination was evaluated by the recognition index (RI): $\mathrm{RI}=T_{N} /\left(T_{N}+T_{F}\right)$.

\section{Accelerating Rotarod test}

Motor coordination and motor learning of mice were assessed by using Rotarod test. The mouse was placed on the roller lane of the Rotarod (Panlab, LE8500, Spain) and started rotating the rod with a steady acceleration from 4 to $40 \mathrm{rpm}$ over a 5 min period. The latency at which the mouse fell off the rod was recorded. Each mouse was given six trials with 10-15 min inter-trial intervals (ITIs).

\section{Forced swim test}

Depression-like behaviors were evaluated through forced swim test. A transparent plastic beaker $(17 \mathrm{~cm}$ in diameter $\times 24 \mathrm{~cm}$ 
A

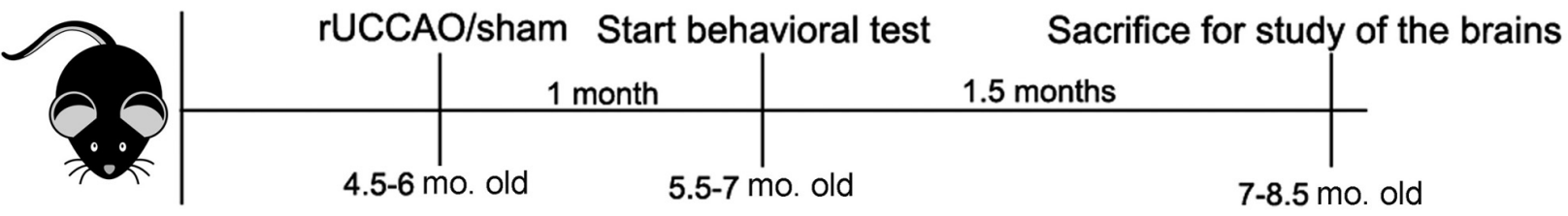

B

Cortex

Hippocampus
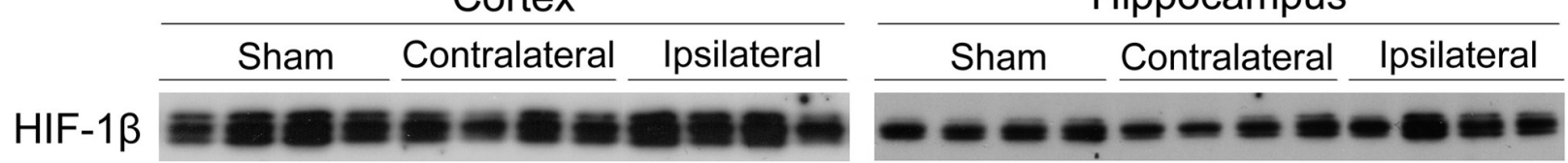

TIGAR
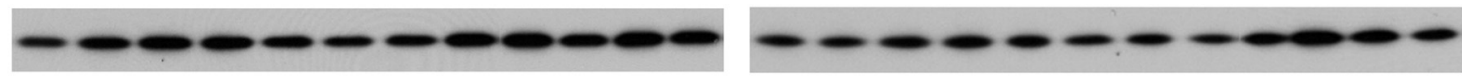

GLUT1

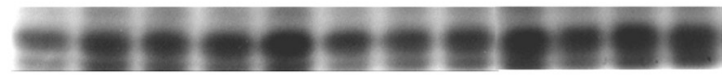

GLUT2
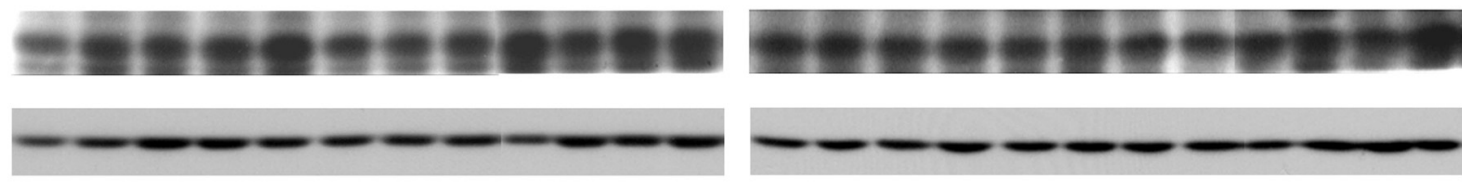

GLUT3
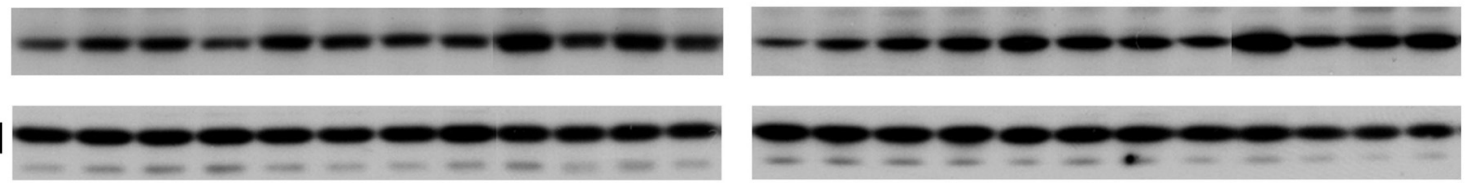

GAPDH

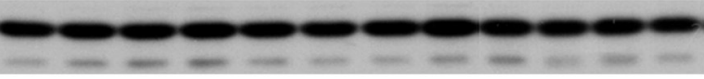

C
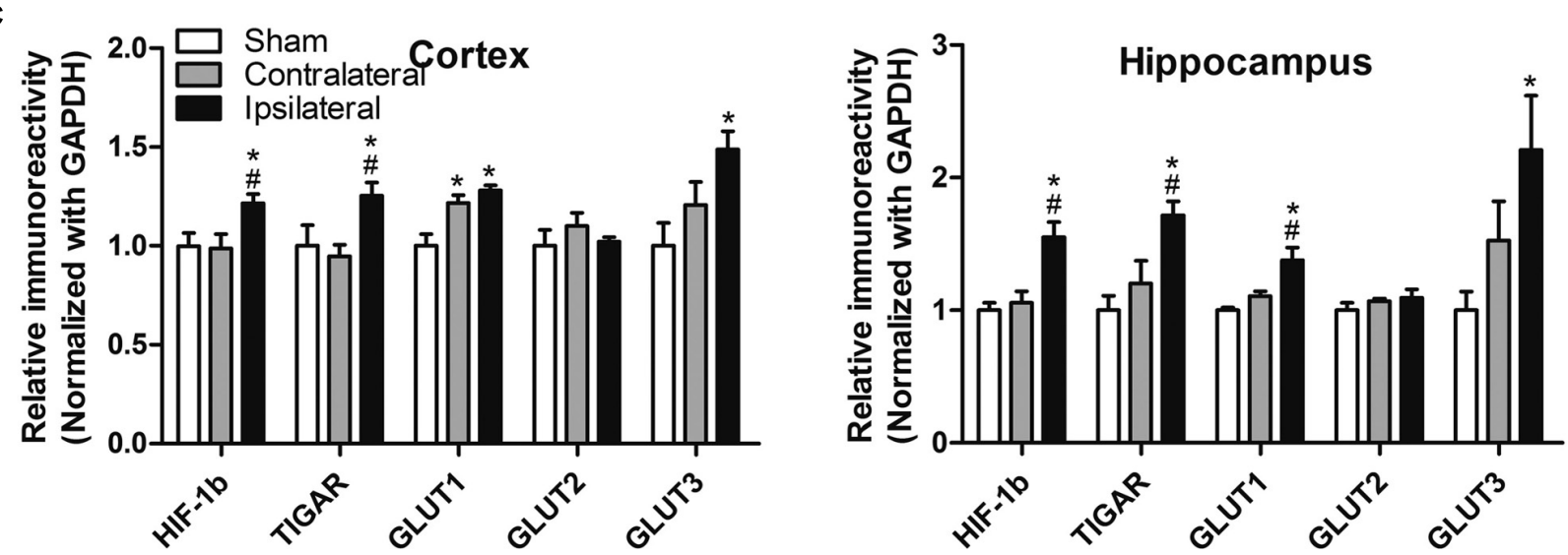

FIGURE 1 | Animal study design (A) and verification of the UCCAO model (B,C). Homogenates of the cortices and hippocampi of mice 2.5 months after sham or UCCAO surgery were analyzed by Western blots developed with the indicated antibodies (B). Each lane represents an individual mouse sample.
The blots were then quantified densitometrically, and the data after normalization with the GAPDH level are presented as mean \pm SEM ( $n=6$ /group), where the values of the sham group are set as 1.0 (C). ${ }^{*} p<0.05$ vs. sham group; $\# p<0.05$ vs. contralateral group. high) containing water $\left(23 \pm 1^{\circ} \mathrm{C}\right)$ to a depth of $17 \mathrm{~cm}$ was used as the swimming apparatus. All mice were individually forced to swim for $6 \mathrm{~min}$, and the duration of immobility was observed and measured during the final $4 \mathrm{~min}$ of the test. Immobility consists of the mouse floating in the water without struggling and only making movements necessary to keep its head above water surface (Slattery and Cryan, 2012).

\section{Footprint test}

Motor coordination and gait were assessed through footprint test using a $50 \mathrm{~cm}$ long and $8 \mathrm{~cm}$ wide runway (with $10 \mathrm{~cm}$ high walls). Before collecting the footprints, each mouse was trained for three or four runs to make sure it was able to run along the runway at a steady pace and in a straight line without wandering. To obtain footprints, the forepaws and hindpaws of the 
mice were respectively coated with red and blue non-toxic paints. To collect the footprints, a fresh sheet of pale white paper was placed on the floor of the runway for each run. The footprint patterns were analyzed for the following four step parameters (all measured in centimeters). (1) Stride length was measured as the average distance of forward movement between each stride. The left/right forelimb and left/right hindlimb stride were also measured separately. (2) Front/hind base width was measured as the average distance between left and right front/hind footprints, respectively. These values were determined by measuring the perpendicular distance of a given step to a line connecting its opposite preceding and proceeding steps. (3) Overlap between forepaw/hindpaw placements were measured as the distance between the front/hind footprints on each side. When the center of the hind footprint fell on top of the center of the preceding front footprint, a value of zero was recorded (Carter et al., 1999).

\section{Morris water maze}

Morris water maze (MWM) was used to evaluate spatial learning and memory of the mice (D'Hooge and De Deyn, 2001). The test was performed in a circular white pool (with a diameter of $180 \mathrm{~cm}$ and a height of $60 \mathrm{~cm}$ ) filled with white dye tinted water and maintained at room temperature $\left(20 \pm 1^{\circ} \mathrm{C}\right)$. The maze was designated of two principal axes with each line bisecting the maze perpendicular to the other one to divide the maze into four equal quadrants. The end of each line demarcates four cardinal points: north $(\mathrm{N})$, south $(\mathrm{S})$, east (E), and west (W). A platform was positioned in the middle of one of the quadrants submerged $1 \mathrm{~cm}$ below water surface. Each mouse performed four trials per day for four consecutive days from semi-random start positions (Table 2) to find the hidden platform. Each trial was terminated as soon as the mouse climbed onto the hidden platform. If a mouse failed to find the platform within $90 \mathrm{~s}$, it was gently guided to it. At the end of each trial, the mouse was left on the platform during a $20 \mathrm{~s}$ ITI,

Table 2 | Mouse start positions in the Morris water maze.

\begin{tabular}{lllll}
\hline Day & Trial 1 & Trial 2 & Trial 3 & Trial 4 \\
\hline Original tests & & & & \\
1 & S & NW & SE & W \\
2 & NW & W & S & SE \\
3 & W & SE & NW & S \\
4 & SE & S & W & NW \\
Probe & NE & & & \\
\hline
\end{tabular}

platform site location: SW

\begin{tabular}{lllll} 
Reversal tests & & & \\
1 & N & E & SW & NW \\
2 & SE & N & NW & W \\
3 & NW & SW & W & N \\
4 & E & NW & N & SE \\
Probe & SW & & & \\
\hline
\end{tabular}

platform site location: NE then dried and returned to its home cage. A 60 s probe test without platform was performed $24 \mathrm{~h}$ after the last trial. The swim path, swim distance $(\mathrm{cm})$, escape latency $(\mathrm{sec})$, swim speed $(\mathrm{cm} / \mathrm{sec})$, time spent in each quadrant ( $\mathrm{sec}$ ), distance traveled in each quadrant $(\mathrm{cm})$, latency to enter the platform site zone $(\mathrm{sec})$, and the number of platform site zone crossings were recorded through an automated tracking system (Smart video tracking system, Panlab; Havard Apparatus). A reversal test was performed on the day after the probe day of original test. For the reversal test, all conditions were the same as the original test except the platform was relocated to the center of the opposite quadrant against the original one.

\section{WESTERN BLOT ANALYSIS}

Mouse brain tissue was homogenized in pre-chilled buffer containing $50 \mathrm{mM}$ Tris- $\mathrm{HCl}(\mathrm{pH} 7.4), 50 \mathrm{mM}$ GlcNAc (inhibitor of O-GlcNAcase that catalyzes the removal of O-GlcNAc from a protein), $20 \mu \mathrm{M}$ UDP (inhibitor of O-GlcNAc transferase), $2.0 \mathrm{mM}$ EGTA, $2 \mathrm{mM} \mathrm{Na}_{3} \mathrm{VO}_{4}, 50 \mathrm{mM} \mathrm{NaF}, 0.5 \mathrm{mM}$ AEBSF, $10 \mu \mathrm{g} / \mathrm{ml}$ aprotinin, $10 \mu \mathrm{g} / \mathrm{ml}$ leupeptin, and $4 \mu \mathrm{g} / \mathrm{ml}$ pepstatin A. Protein concentrations of the homogenates were determined by using Pierce ${ }^{\mathrm{TM}} 660 \mathrm{~nm}$ Protein Assay kit (Thermo Fisher Scientific Inc.). The samples were resolved in $10 \%$ or $12.5 \%$ sodium dodecyl sulfate polyacrylamide gel electrophoresis (SDS-PAGE) and electro-transferred onto Immobilon-P membrane (Millipore, Bedford, MA, USA). The blots were then probed with primary antibody (Table 1) and developed with the corresponding horseradish peroxidase-conjugated secondary antibody and ECL kit (Pierce, Rockford, IL, USA). Densitometrical quantification of protein bands in Western blots were analyzed by using the Multi Gauge V3.0 software (Fuji Photo Film Co., Ltd).

\section{IMMUNO-DOT-BLOT ASSAY}

The level of O-GlcNAcylation in the brain homogenates was determined by using a quantitative immuno-dot-blot assay (Liu et al., 2002) with modifications. Briefly, homogenate samples were first dissolved in Laemmli buffer and then diluted with the homogenizing buffer till the SDS concentration was $0.05 \%$ or lower. The diluted samples were dotted onto nitrocellulose membrane (Schleicher and Schuell, Keene, NH, USA) at $6 \mathrm{ul}$ per grid of $6 \times 6 \mathrm{~mm}$ in size. The blots were placed in a $37^{\circ} \mathrm{C}$ oven for $0.5-1 \mathrm{~h}$ to allow the protein binding to the membrane. After the membrane was blocked with TBST $(10 \mathrm{mM}$ Tris-HCl, pH7.5, $150 \mathrm{mM} \mathrm{NaCl}, 0.3 \%$ Tween-20) for $1 \mathrm{~h}$, the blots were overlaid with a mixture of antibodies RL-2 and CTD110.6 and then incubated at $4^{\circ} \mathrm{C}$ overnight. After washing with TBST, the blots were processed as described above for Western blots.

\section{IMMUNOHISTOCHEMICAL STAINING}

Floating sagittal sections were incubated at room temperature in $0.3 \% \mathrm{H}_{2} \mathrm{O}_{2}$ for $30 \mathrm{~min}$ and then in $0.3 \%$ Triton $\mathrm{X}-100$ for $15 \mathrm{~min}$, washed in PBS, and blocked in a solution containing 5\% normal goat serum and $0.1 \%$ Triton X-100 for $30 \mathrm{~min}$. Sections were then incubated at $4^{\circ} \mathrm{C}$ with primary antibody overnight, followed by development with biotinylated secondary antibody and 
avidin/biotinylated horseradish peroxidase (Santa Cruz Biotechnology). The sections were stained with peroxidase substrate and then mounted on microscope slides (Brain Research Laboratories, Newton, MA, USA), dehydrated, and covered with coverslips.

\section{FLUORO-JADE STAINING}

Floating sagittal sections were mounted on gelatin coated slide, dried at room temperature overnight, and then rinsed in distilled water for $1 \mathrm{~min}$. After incubation in freshly prepared $0.06 \%$ potassium permanganate for $10 \mathrm{~min}$ and washes with distilled water, sections were stained with $0.0004 \%$ Fluoro-Jade B in $0.09 \%$ acetic acid for $10 \mathrm{~min}$. After washes again, the sections were further stained with DAPI. Stained sections were observed under a fluorescence microscope (PCM2000, Nikon, Melville, NY, USA), and positive cells were counted under $20 \times$ magnification by the use of Image J software (National Institutes of Health).

\section{STATISTICAL ANALYSIS}

For behavioral tests, data were analyzed by two-way ANOVA or unpaired two-tailed $t$-test using Graphpad Prism 5s. For biochemical analyses, data were analyzed by one-way ANOVA followed by Tukey's post hoc tests or unpaired two-tailed $t$ tests, using Graphpad Prism 5. All data are presented as means \pm SEM, and $p<0.05$ was considered statistically significant.

\section{RESULTS}

\section{VERIFICATION OF THE CCH MODEL}

Previous studies have demonstrated that UCCAO does not produce histologically detectable cerebral ischemia or neuronal cell death (Kitagawa et al., 2005; Lee et al., 2011; Pimentel-Coelho et al., 2013). Thus, we first verified the success of UCCAO by detecting the levels of hypoxia inducible factor- $1 \beta$ (HIF-1 $\beta$ ), TP53induced glycolysis and apoptosis regulator (TIGAR) and glucose transporters (GLUTs) 1 and 3, because the expression of these proteins increases during cerebral or cardiac ischemia (Watanabe et al., 2009; Kimata et al., 2010; Chan et al., 2011; Iwabuchi and Kawahara, 2011; Yan et al., 2011; Yuan et al., 2011; Hoshino et al., 2012; Wang et al., 2012a,b). As expected, we found significant increases in the levels of these proteins in both the cerebral cortex and the hippocampus of the ipsilateral side of the UCCAO mice (Figures 1B,C). A trend of increase in GLUT1 and GLUT3 was also seen in the contralateral side, and this increase reached statistical significance for cerebrocortical GLUT1 (Figure 1C), which might suggest that there was mild chronic hypoperfusion at the contralateral hemisphere in the mice 2.5 months after UCCAO. As a negative control, the level of GLUT2 was not altered after UCCAO (Figures 1B,C). These results showed the success of $\mathrm{CCH}$ in the brains of mice after UCCAO.

\section{CCH CAUSES ANXIETY AND DEFICITS OF MOTOR FUNCTIONS}

To investigate the effects of $\mathrm{CCH}$ on the mouse behavior, we first monitored general conditions of the mice, including body weight and food intake after sham or $\mathrm{CCH}$ surgery. We found that the body weights of both groups decreased one week after the surgery (Figure 2A), indicating the consequence of stress from the surgery itself on the mice. The body weight recovered a week later, but the $\mathrm{CCH}$ mice constantly had a significantly higher body weights (5-6\% higher) than the sham mice. Interestingly, the $\mathrm{CCH}$ mice had a much lower food intake ( $\sim 20 \%$ lower) than the sham mice after the marked decline during the first week after the surgery (Figure 2B). The surprising opposite changes of the body weight and food intake of the $\mathrm{CCH}$ mice suggest that $\mathrm{CCH}$ might have caused decreased metabolism and/or activity in mice.

We then assessed the general behaviors including exploratory activity, anxiety, and locomotor activity of the mice. By testing the mice in an open field, we found that the $\mathrm{CCH}$ mice traveled a shorter distance (Figure 2C) and spent less time (data not shown) in the central area of the open field than the sham mice. In an elevated plus maze for measuring open space- and height-induced anxiety, we found that the $\mathrm{CCH}$ mice entered the CAs more frequently (Figure 2D). Together, these results suggest an increased level of anxiety and a decreased level of spontaneous exploratory activity of the $\mathrm{CCH}$ mice, the latter of which is consistent with the increased body weights and decreased food intake of the $\mathrm{CCH}$ mice observed above.

To study whether UCCAO changes the gait and motor coordination of the mice, we carried out foot print test and Rotarod test. We found that the left forelimb stride of the $\mathrm{CCH}$ mice was slightly shorter than that of the sham mice as measured in the footprint test (Figure 2E). However, the decrease in the left forelimb stride of the $\mathrm{CCH}$ mice was not statistically significant as compared to the right forelimb stride of the same mice. The Rotarod test showed marked decrease of the fall latency of the $\mathrm{CCH}$ mice as compared to the sham group (Figure 2F). These results indicate that the $\mathrm{CCH}$ mice might have a slight decrease in motor activity of left side (contralateral side) and a pronounced decrease in motor coordination and motor learning. However, forced swim test did not show any detectable abnormality in swimming, nor the time spent immobile in the $\mathrm{CCH}$ mice (Figure 2G), indicating that the $\mathrm{CCH}$ mice were not under a depressive state.

\section{CCH LEADS TO MILD IMPAIRMENT IN MEMORY}

The non-spatial working memory related to frontal subcortical circuits of the mice was assessed by using object recognition test (Sarti et al., 2002) with $15 \mathrm{~min}$ and $24 \mathrm{~h}$ intervals between the sample phase and the test phase. During the sample phase, all mice spent the same amount of time in exploring both objects (Figure 2H). However, the $\mathrm{CCH}$ mice spent less time exploring the novel object than the sham mice when tested both $15 \mathrm{~min}$ and $24 \mathrm{~h}$ after the sample phase, although the decrease only at the 15 min time point reached the statistical significance (Figure 2I). These results indicate a short-term memory deficit in the $\mathrm{CCH}$ mice.

We also employed MWM to investigate the long-term spatial reference learning and memory of these mice. We observed a slight but significant decrease in swim speed of the $\mathrm{CCH}$ mice during the training phase (Figure $\mathbf{3 A}$ ), which is consistent with the deficit in locomotor activity of these mice, as detected above. During the training phase in the water maze, both groups showed proper learning as evidenced by continuous decrease in the distance traveled (Figure 3B) and the latency (data not shown) to locate the hidden escape platform. Retrieval of spatial memory was more specifically tested with the probe trial performed $24 \mathrm{~h}$ after the last 
A
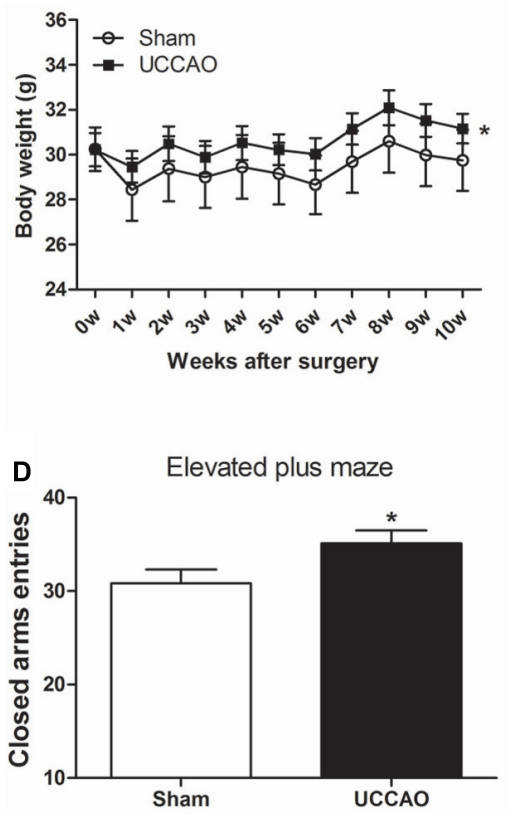

G

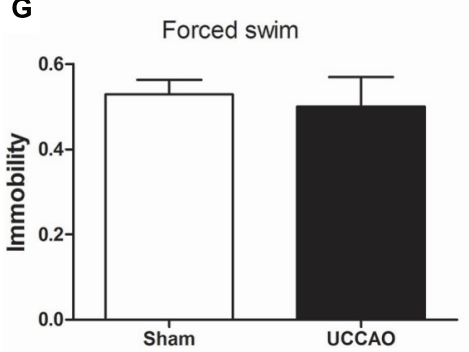

B

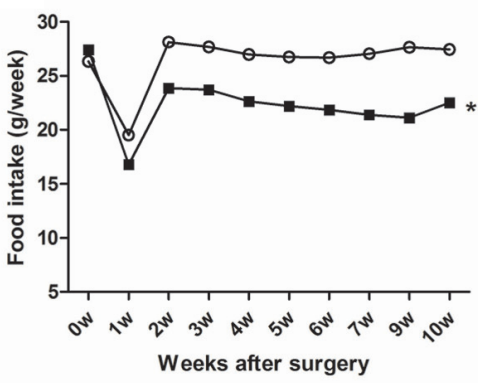

E
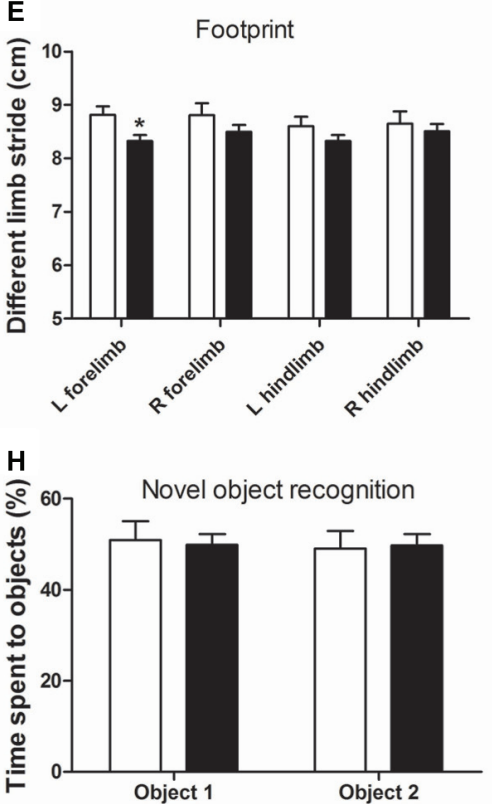

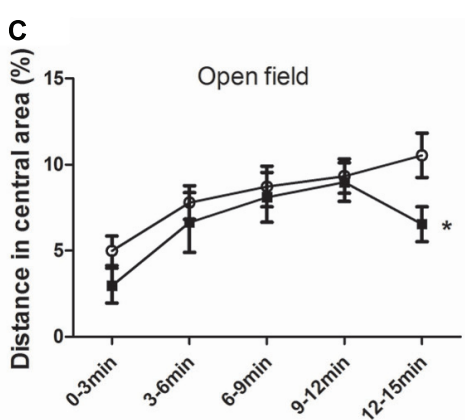

$\mathbf{F}$
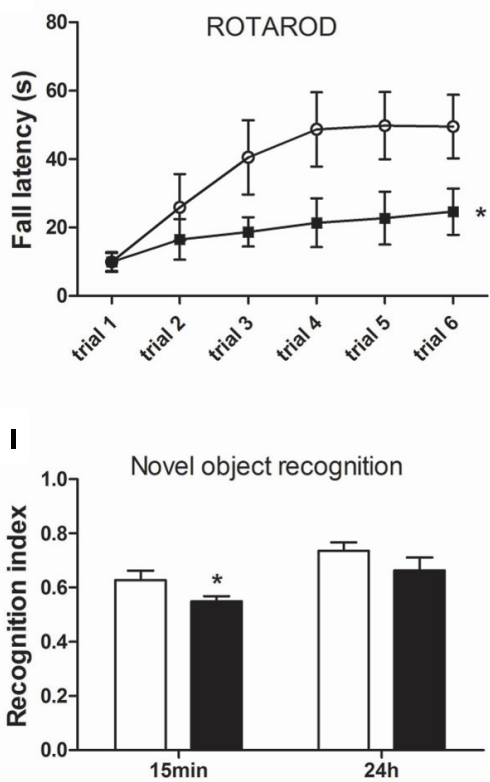

FIGURE 2 | General behavior and short-term memory of mice with $\mathbf{C C H}$. The body weight and food intake were monitored once a week after sham or UCCAO surgery (A,B). Spontaneous locomotor and exploratory activity was assessed in an open field (C). Anxiety-like behaviors were evaluated in an elevated plus maze, and the CAs entries are shown (D). Gait was evaluated using footprint test, and different limb strides are shown (E). Motor

coordination and balance were evaluated using accelerating Rotarod, and the fall latency of each trial is shown (F). Depression was evaluated using forced swim test, and last 4 min immobility out of total 6 min is shown (G). One-trial object recognition task was carried out in an open field. Time spent exploring two identical objects during sample phase are shown as percentage of object exploring time (H). Object discrimination during test phase is presented by the recognition index (time exploring the novel object/total time for exploring) (I). Data are reported as mean \pm SEM. ${ }^{*} p<0.05$ vs. sham mice. training trial. Memory performance of the mice was determined by the number of the former platform site crossings and the percentage of time/distance animals spent/traveled in the target quadrant. We found that the $\mathrm{CCH}$ mice showed a decrease in the number of platform crossings (Figure 3C), a slight increase of the latency to first crossing (data not shown), and slightly less preference toward the target quadrant (Figure 3D). However, none of these changes observed in the $\mathrm{CCH}$ mice reached a statistical difference. These results suggest that the $\mathrm{CCH}$ mice did not have a deficit in spatial memory or the tests and/or the sample size we used did not have sufficient power to detect the mild deficit of spatial memory in the $\mathrm{CCH}$ mice.

In an attempt to increase the sensitivity of the spatial memory test, we further carried out five days' reversal test in the MWM starting on the next day following the probe test of the original test.
For the reversal test, the escape platform was relocated to the quadrant opposite to the original quadrant. We observed that the $\mathrm{CCH}$ mice again had a lower swim speed (Figure 3E), a marked decrease in the mean platform site crossing (Figure 3G) and slightly less preference toward the target quadrant (Figure $3 \mathbf{H}$ ) at the probe trial of the reversal test. However, these changes did not reach statistical significance, apparently due to large scattering of the data. The training curve of the reversal test indicated that the $\mathrm{CCH}$ mice traveled a shorter distance (Figure 3F) and had a shorter latency (data not shown) to locate the new hidden escape platform during the first three days of the training phase. To learn if this was because the $\mathrm{CCH}$ mice remembered the previous escape less well than the sham mice, we analyzed the distance traveled and the time spent in the quadrant where the previous old hidden escape platform was located. We found that the $\mathrm{CCH}$ mice traveled a 

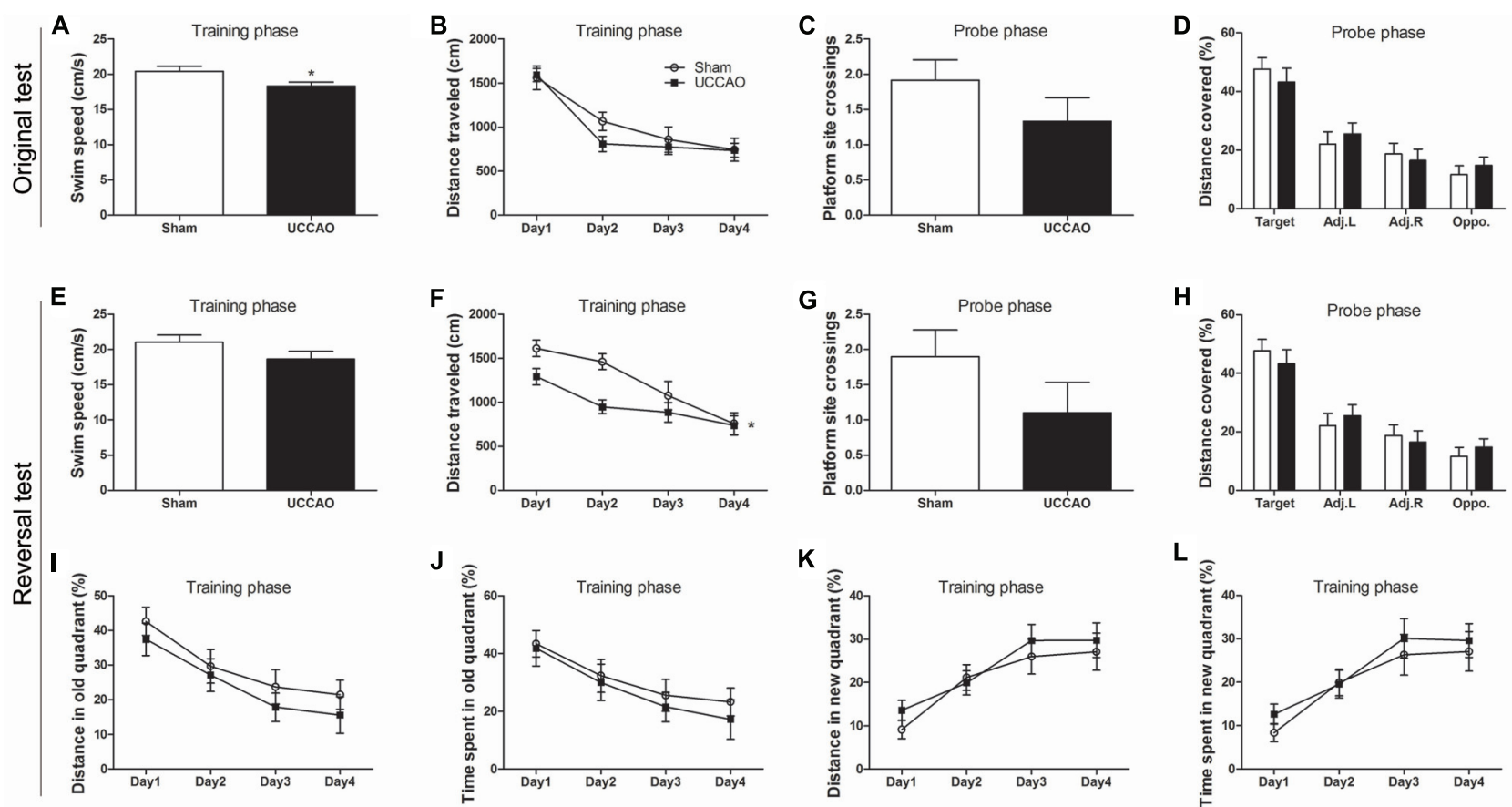

FIGURE 3 | Morris water maze tests of mice with CCH. Spatial memory of the mice was tested in the Morris water maze test (A-D) and the consecutive reversal test $(\mathbf{E}-\mathbf{L})$. The average swim speed in the training phase $(\mathbf{A}, \mathbf{E})$, the distance traveled to the hidden platform during training $(\mathbf{B}, \mathbf{F})$, the number of the platform site crossings during the probe trial $(\mathbf{C}, \mathbf{G})$, the percentage of shorter distance (Figure 3I) and spent shorter time (Figure 3J) in that quadrant than the sham mice, suggesting that the apparently better performance of the $\mathrm{CCH}$ mice during the first three days' training in the reversal water maze, as seen in Figure 3F, was actually because these mice remembered the old platform location less well than the sham mice. The $\mathrm{CCH}$ and the sham mice showed no difference in the distance traveled (Figure 3K) and the time spent (Figure 3L) in the quadrant where the new escape platform was located during the training phase of the reversal water maze test, again suggesting no learning deficit of the $\mathrm{CCH}$ mice. Taken together, these results suggest that the $\mathrm{CCH}$ mice had no impairment in learning, but had a slight impairment in long-term spatial memory.

\section{CCH LEADS TO DECREASED O-GLCNACYLATION AND INCREASED TAU PHOSPHORYLATION}

Because $\mathrm{CCH}$ is expected to reduce brain glucose metabolism and O-GlcNAcylation is a sensor of intracellular glucose metabolism (Zachara and Hart, 2004) and regulates tau phosphorylation negatively (Liu etal., 2004, 2009; Li etal., 2006), we investigated protein O-GlcNAcylation and its regulating enzymes O-GlcNAc transferase (OGT, which adds GlcNAc groups to proteins) and O-GlcNAcase (OGA, which removes GlcNAc from proteins), as well as tau phosphorylation. We found a significant decrease in the O-GlcNAcylation level in both the cortex and the hippocampus of the ipsilateral side of the $\mathrm{CCH}$ mice, as determined by immuno-dot blots developed with a distance traveled in the target, adjacent left (Adj.L), adjacent right (Adj.R), and opposite (Opp) quadrants during probe trial $(\mathbf{D}, \mathbf{H})$ and the gradual reduction in percent time/distance in the quadrant of previous platform and the gradual shift to the new quadrant (I-L) are shown. Data are reported as mean \pm SEM. ${ }^{*} p<0.05$ vs. sham mice. mixture of two monoclonal antibodies both against various $\mathrm{O}$ GlcNAcylated proteins (Figures 4A,B). To uncover the underlying mechanism for the change of O-GlcNAcylation, we determined OGT and OGA levels by Western blots and observed significant decrease in the OGT level in the hippocampus of the $\mathrm{CCH}$ mice (Figures 4A,B), which might partially explain the decreased O-GlcNAcylation.

Site-specific tau phosphorylation in the mouse brains was determined by Western blot developed with several phosphorylation-dependent and site-specific tau antibodies, and the total tau by a polyclonal pan-tau antibody R134d. We found that tau phosphorylation increased markedly at most of the phosphorylation sites studied in the hippocampus and at Thr212 and Ser262 of the cortex of the CCH mice (Figures 4C,D), which is consistent with the decrease of O-GlcNAcylation. Surprisingly, the increased tau phosphorylation was also seen at the contralateral side, where O-GlcNAcylation was not found to be decreased, of the UCCAO mice, suggesting that additional mechanism might be involved in tau phosphorylation at the contralateral side of the UCCAO mice.

\section{CCH DYSREGULATES SYNAPTIC PROTEINS}

Synapses are the structural basis of memory and cognition, which depends on synaptic plasticity that is in turn regulated by modulation of neurotransmitter release from the pre-synaptic site and of the number, types and properties of neurotransmitter receptors at the post-synaptic site. To understand the molecular basis 

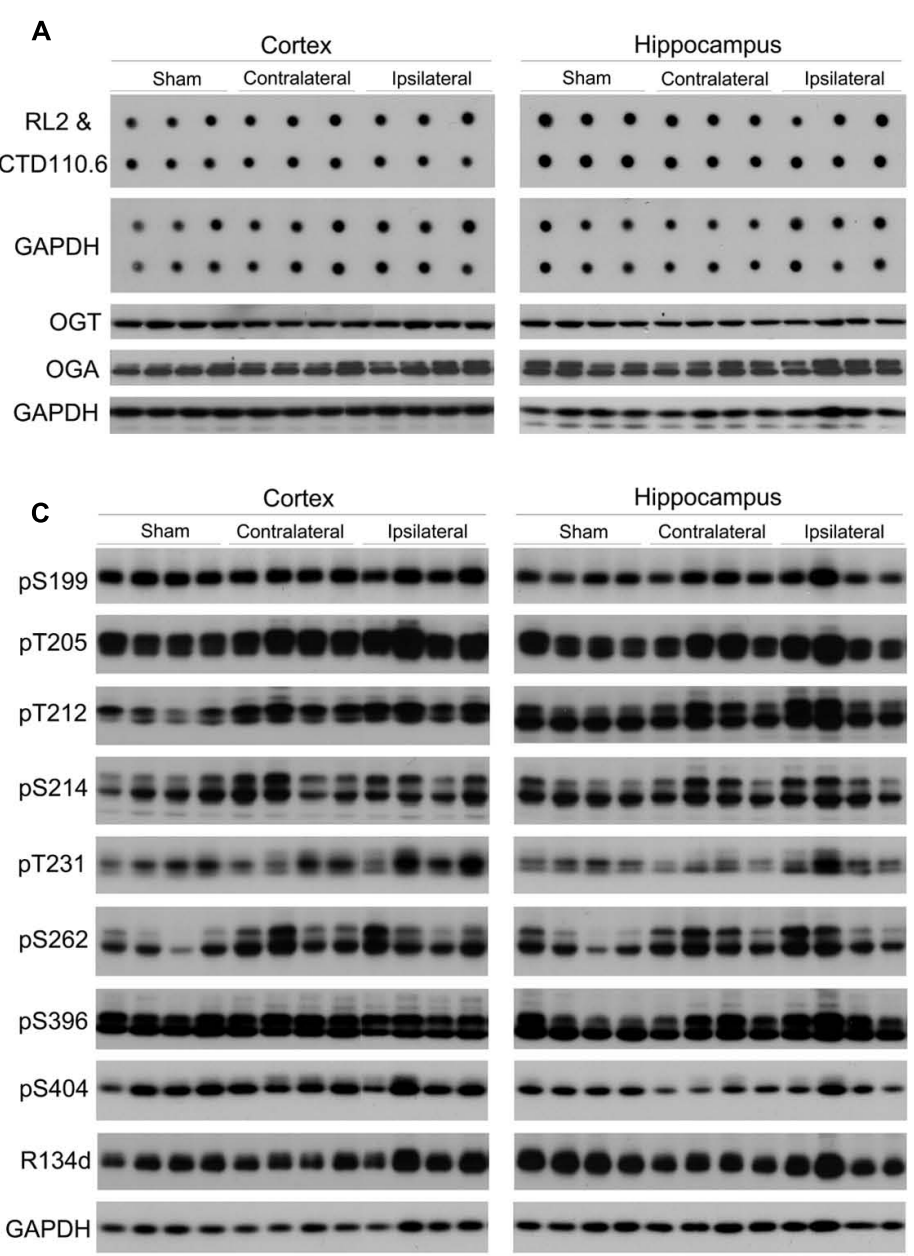

B
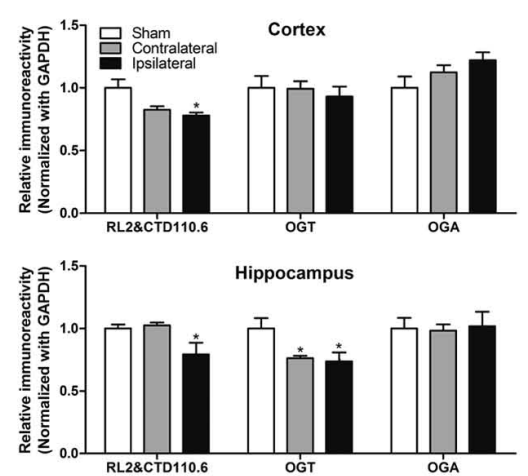

D
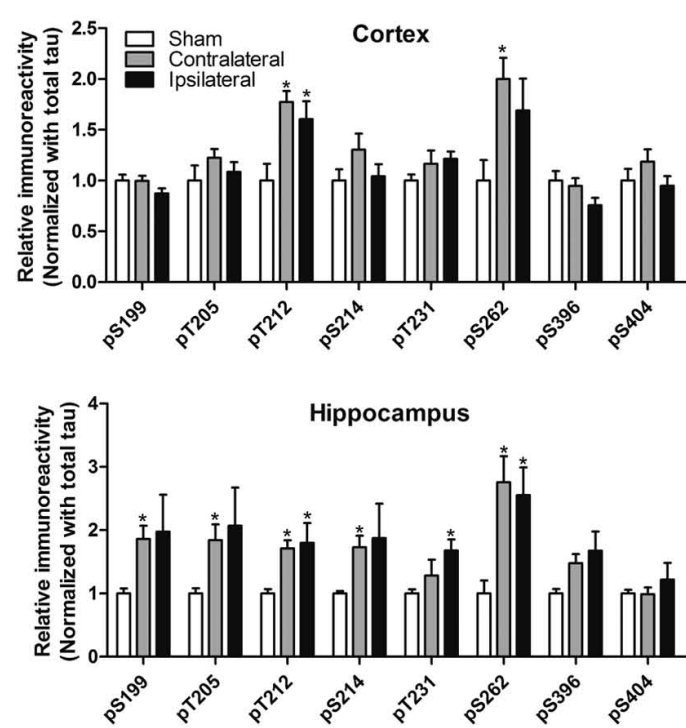

FIGURE 4 | Effects of $\mathbf{C C H}$ on O-GIcNAcylation and tau

phosphorylation. Homogenates of the cortices and hippocampi of mice 2.5 months after sham or UCCAO surgery were analyzed by immuno-dot blots developed with a mixture of monoclonal antibodies RL2 and

CTD110.6 against O-GlcNAcylated proteins and Western blots developed with the indicated antibodies $(\mathbf{A}, \mathbf{C})$. The blots were then quantified densitometrically, and the data after normalization with the GAPDH (B) or R134d for total tau level (D) are presented as mean \pm SEM ( $n=6$ /group), where the values of the sham group are set as 1.0. ${ }^{*} p<0.05$ vs. sham group. of the mild memory impairment we detected in the $\mathrm{CCH}$ mice, we determined the levels of major pre- and post-synaptic proteins in the mouse brains. We found a dramatic increase in the level of synapsin but marked decrease in synaptophysin, both of which are commonly used as pre-synaptic markers, in both the cerebral cortex and the hippocampus of the $\mathrm{CCH}$ mice (Figures 5A,B). The level of post-synaptic density 95 (PSD95), a post-synaptic marker, was also found to be increased in the cerebral cortex of the $\mathrm{CCH}$ mice. These results indicate a dysregulation of synaptic proteins in the brains of the $\mathrm{CCH}$ mice. Interestingly, the contralateral side of the $\mathrm{CCH}$ mice tended to have the similar dysregulation of synaptic proteins, implicating that milder but similar changes might occur at the contralateral side of the $\mathrm{CCH}$ mice.

We also carried out immunohistochemical studies of synaptophysin in the mouse brains. The decreased immunostaining was seen throughout the cerebral cortex and various sectors of the hippocampus, more apparent in the ipsilateral side (Figure 5C). These results are consistent with the results from Western blots.

\section{CCH AFFECTS THE HBP AND INSULIN SIGNALING PATHWAY IN THE BRAIN}

A small fraction of intracellular glucose is metabolized into uridine diphosphate- $\mathrm{N}$-acetylglucosamine (UDP-GlcNAc), the donor of protein O-GlcNAcylation, through the HBP, and OGT activity is dependent on intracellular UDP-GlcNAc level (Hanover etal., 2010). The rate limiting enzyme of the HBP is L-glutamine:fructose-6-phosphate amidotransferase (GFAT). As we had observed a decreased level of O-GlcNAcylation after $\mathrm{CCH}$, we further investigated the level of GFAT2, the major isoform of GFAT in the mammalian brain, and found that the GFAT2 level was increased in the ipsilateral hippocampus, but not in the cerebral cortex, of the CCH mice (Figures 6A,B).

Glucose metabolism as well as neural plasticity and memory are modulated by insulin signaling (Gerozissis, 2008; Cardoso et al., 2009), which is deregulated in AD brain (Liu et al., 2011). The classical insulin signaling pathway includes insulin receptor (IR), insulin-like growth factor-1 receptor (IGF-1R), insulin receptor 
A

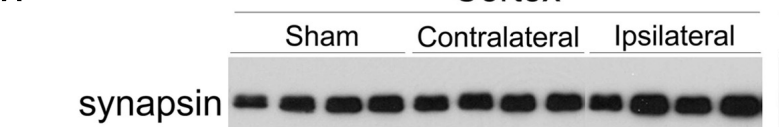

synaptophysin

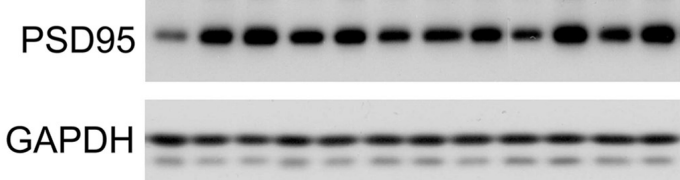

Hippocampus

Sham Contralateral Ipsilateral
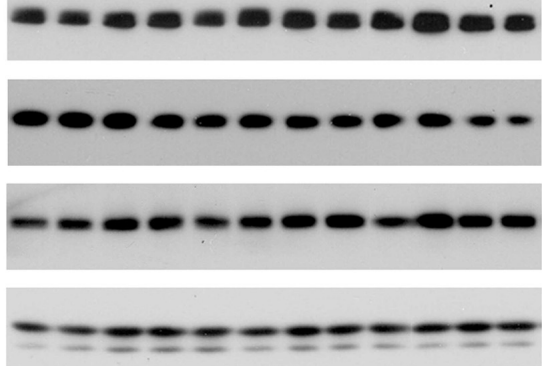

B

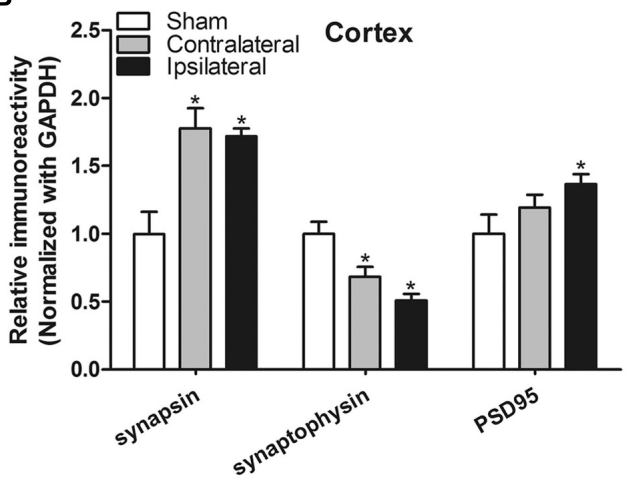

C

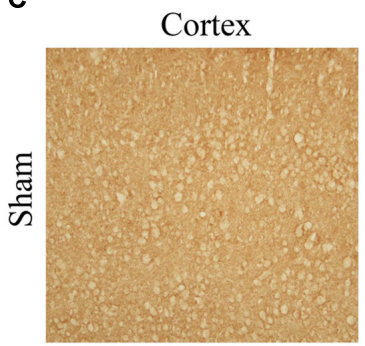

CA1
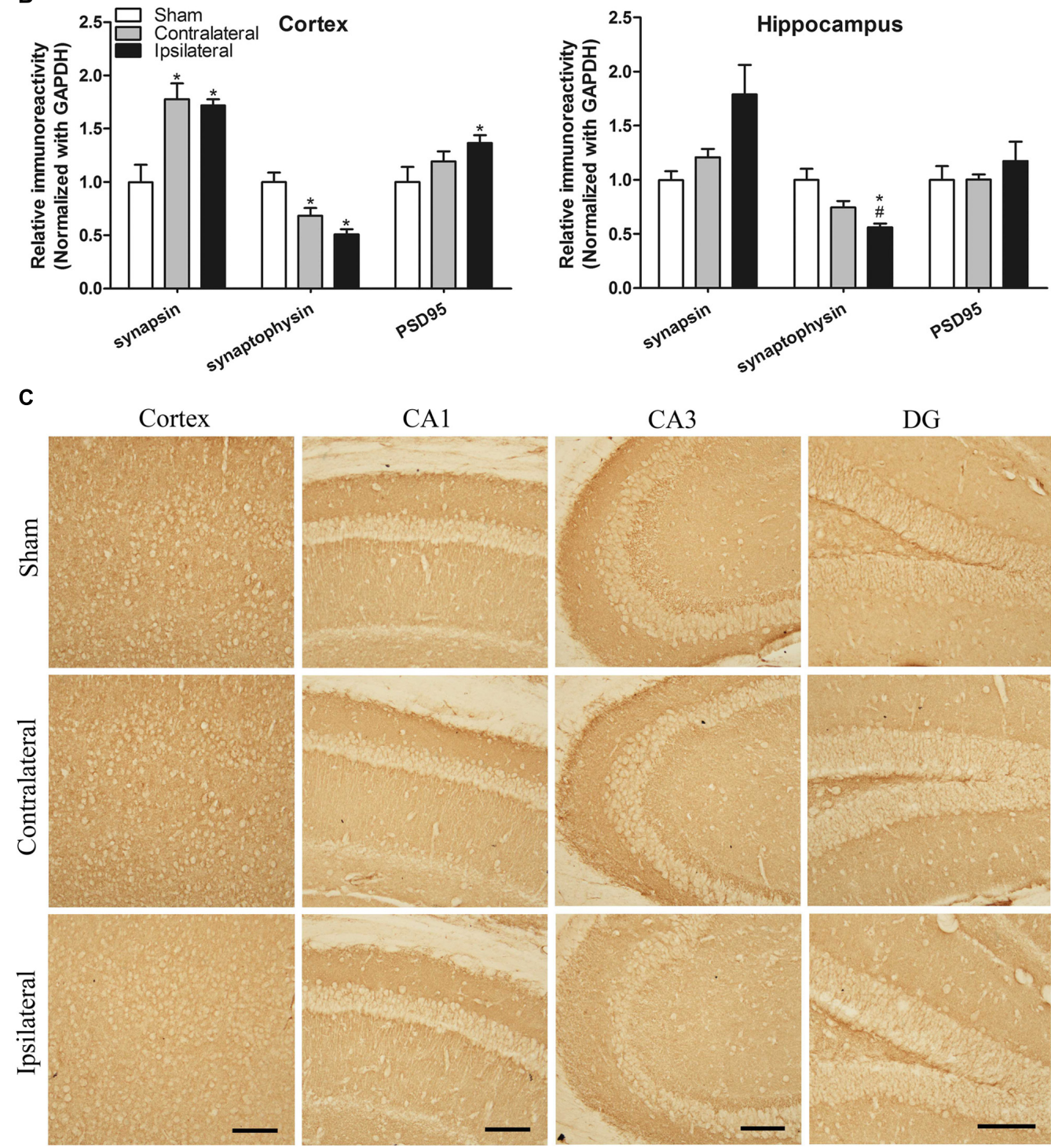

FIGURE 5 | Effects of $\mathbf{C C H}$ on synaptic proteins. Homogenates of the cortices and hippocampi of mice 2.5 months after sham or UCCAO surgery were analyzed by Western blots developed with the indicated antibodies (A). The blots were then quantified densitometrically, and the data after normalization with the GAPDH level are presented as

mean \pm SEM ( $n=6 /$ group), where the values of the sham group are set as 1.0 (B). ${ }^{*} p<0.05$ vs. sham group. $\# p<0.05$ vs. contralateral group. The coronal brain sections were immunostained with antibody against synaptophysin (C). CA, Cornu Ammonis; DG, dentate gyrus; Scale bar: $100 \mu \mathrm{m}$. 


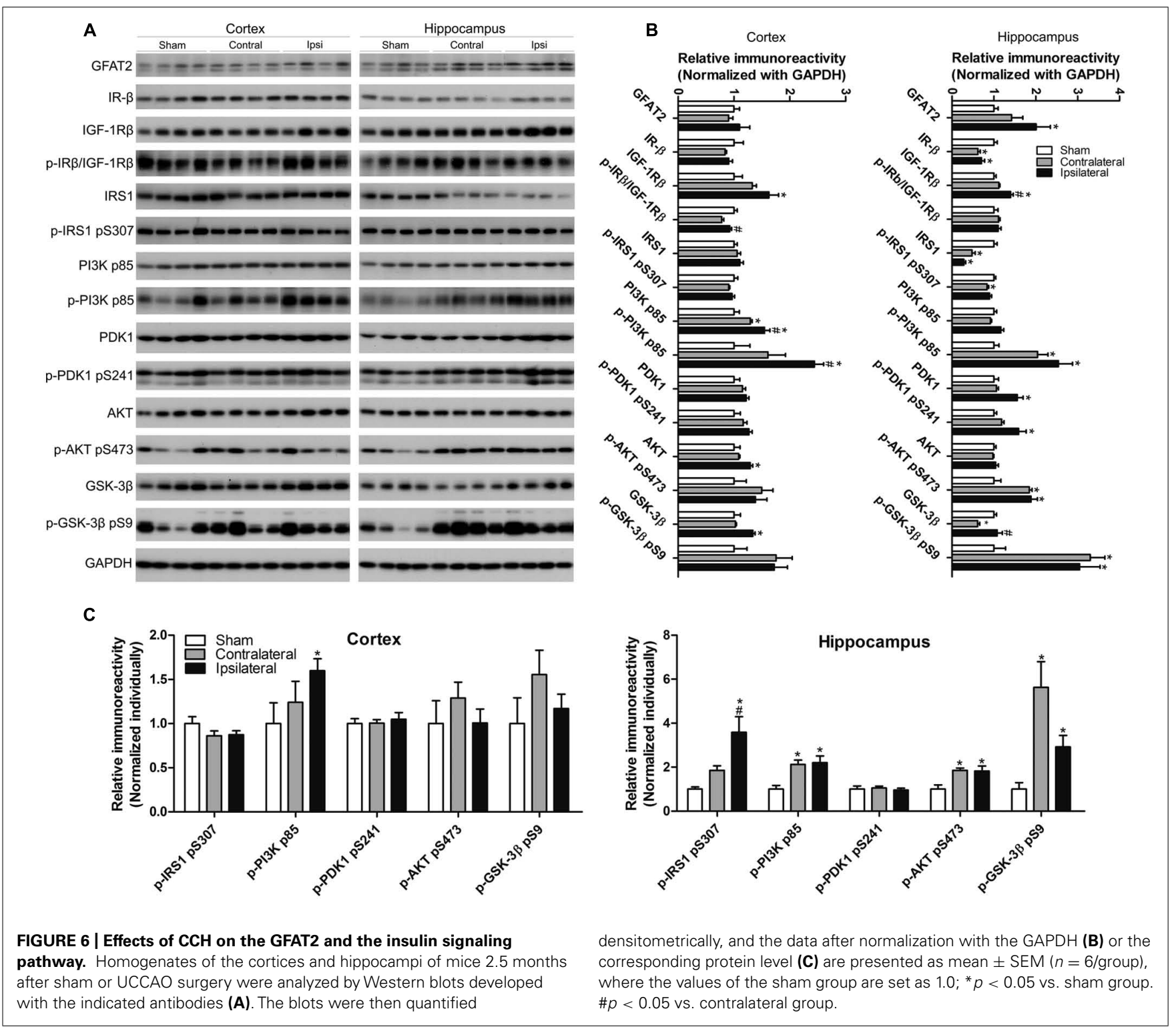

substrate-1 (IRS-1), phosphatidylinositide 3-kinases (PI3K), 3phosphoinositide-dependent protein kinase-1 (PDK1), protein kinase B (AKT), and glycogen synthase kinase-3 (GSK-3). This signaling is activated through the phosphorylation of each component by its upstream component of the signaling cascade. We therefore studied the brain insulin signaling by determining the level and activation status of each component of the insulin signaling pathway in the brain by quantitative western blots. The activation status was estimated by determining the level of phosphorylation, which reflects the enzymatic activity, with the phosphorylation-dependent antibodies. We observed changes of the level and/or phosphorylation of several components of the insulin signaling pathway in the ipsilateral side of the brains of UCCAO mice (Figure 6). These changes included increases in the levels of IGF-1R $\beta$, the p85 subunit of PI3K and its phosphorylation, PDK1 and its phosphorylation, and the phosphorylation of AKT and GSK-3 $\beta$, as well as decreases in the levels

of IR- $\beta$ and IRS1 phosphorylation in the hippocampus. In case of the levels of IR- $\beta$, IRS1 and the phosphorylation of PI3K $\mathrm{p} 85$, AKT, and GSK-3 $\beta$, the UCCAO-induced alterations were also seen in the contralateral side of the brain. The changes of insulin signaling in the cerebral cortex were somewhat similar but not identical to those seen in the hippocampus. Taken together, these results indicate that $\mathrm{CCH}$ results in alterations in insulin signaling.

\section{CCH LEADS TO NEURONAL DEGENERATION}

To investigate whether $\mathrm{CCH}$ for 2.5 months causes neuronal degeneration, we stained the mouse brain frozen sections with Fluoro-Jade that labels degenerating neurons with green fluorescence. Overall, there were very few Fluoro-Jade positive neurons in the control mouse brain. We did not observe widespread Fluoro-Jade positive staining throughout the brain with $\mathrm{CCH}$ either. However, we found a slight increase in the number of 


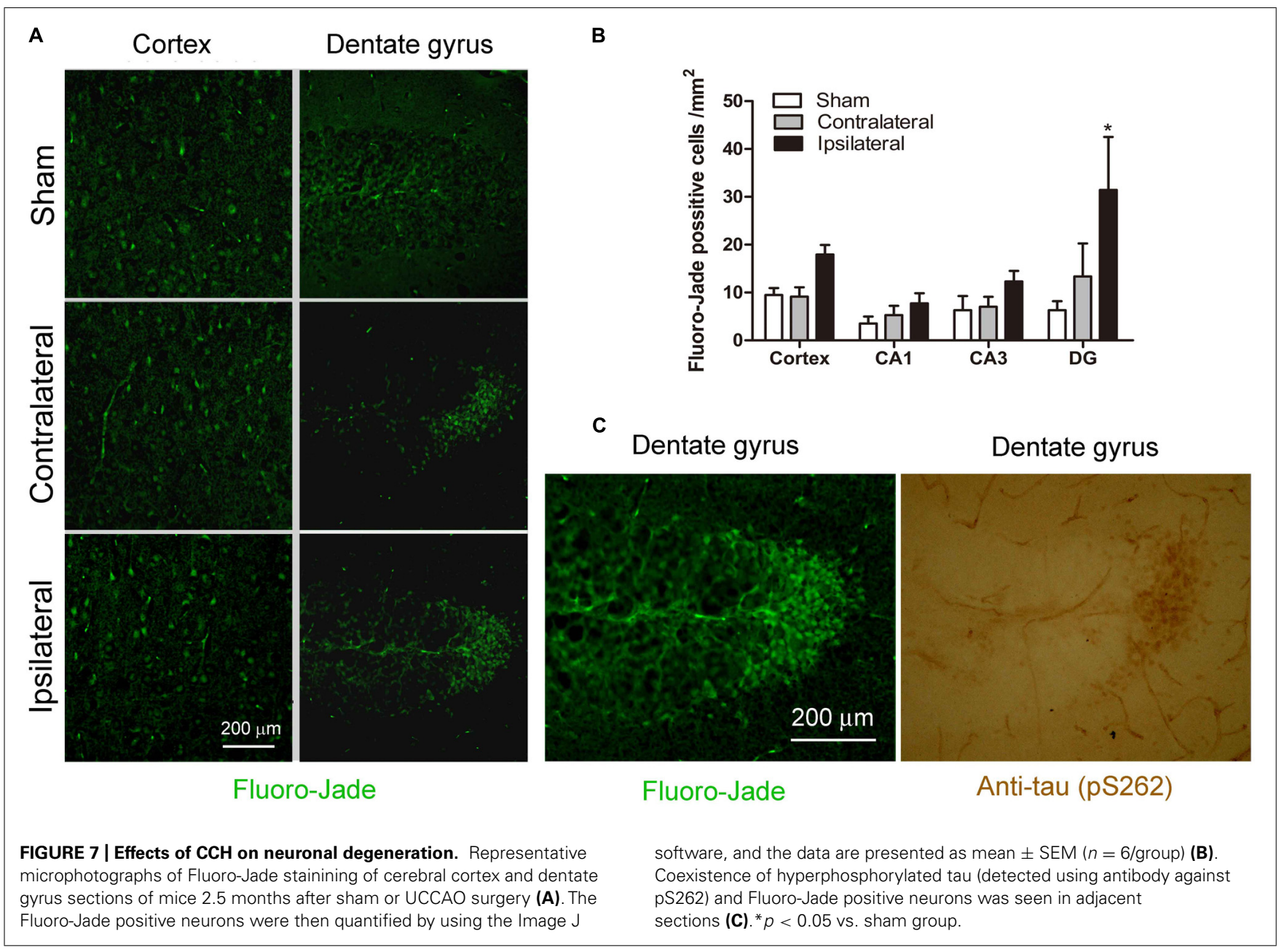

Fluoro-Jade positive neurons in the ipsilateral cerebral cortex and hippocampus of the UCCAO brains, and this increase was most obviously seen in the granule cells of the crest of the dentate gyrus (Figure 7A). Quantification of the Fluoro-Jade positive neurons showed a significant increase in the ipsilateral side of cerebral cortex and dentate gyrus (Figure 7B). These results indicate that $\mathrm{CCH}$ for 2.5 months induces mild neurodegeneration and the dentate gyrus is the most sensitive area for $\mathrm{CCH}$-induced neurodegeneration.

To investigate whether the $\mathrm{CCH}$-induced chronic neurodegeneration is associated with tau hyperphosphorylation, we stained the adjacent brain sections with anti-pS262 that detects tau phosphorylated at Ser 262 and this immunoreactivity was increased dramatically in the $\mathrm{CCH}$ brains (Figure 4). We found a marked increase in anti-pS262 staining in neurons that are Fluoro-Jade positive in the dentate gyrus (Figure 7C). These observations suggest that the $\mathrm{CCH}$-induced neuronal degeneration might be caused or associated with abnormal hyperphosphorylation of tau.

\section{DISCUSSION}

The human brain constitutes only $\sim 2 \%$ of the body's mass, but it receives 15-20\% of the body's blood supply and accounts for up to $50 \%$ of the total body glucose utilization under the basal condition (Peters, 2011). Normal brain function requires a steady blood supply to maintain the stable energy state carrying out all of its cellular and molecular needs. When the blood supply to the brain becomes suboptimal for a long period of time, clinical implications involving chronic hypoperfusion and a variety of brain changes may occur. A series of experiments designed to mimic $\mathrm{CCH}$ have been achieved by permanent or reversible occlusion of two or three major vessels supplying the brain in rats. UCCAO is one of the approaches to produce $\mathrm{CCH}$ in mice and results in a $35-55 \%$ decrease of the ipsilateral cortical perfusion, as detected 28 days after the UCCAO surgery (Kitagawa et al., 2005). This model is thus ideal to investigate the contribution of $\mathrm{CCH}$ to cognitive deficits and $\mathrm{AD}$ pathology.

Glucose is the primary source of fuel for energy-demanding activities in mammalian brain. Together with oxygen, glucose is delivered by the circulation for the metabolic chores that keep brain cells healthy and functioning (Erecińska and Silver, 1989; Qutub and Hunt, 2005). The transport of glucose from blood into neurons requires GLUTs. Glucose is first transported from bloodstream into extracellular space through GLUT1, which is highly expressed in the endothelial cells of the blood-brain barrier, 
then further transported into neurons through neuronal GLUT3. As a response to the energy-demand following cerebral hypoperfusion, the expression and activity of these glucose transporters increase (Vannucci et al., 1997, 1998; Yu et al., 2008). This compensation is mainly mediated by the transcription factor HIF-1 that responds to hypoxia (Singh et al., 2012). TIGAR is a recently discovered enzyme that primarily functions as a regulator of glucose metabolism in the cell (Bensaad et al., 2006). Its expression is also elevated in responding to cardiac ischemia (Kimata et al., 2010). In the present study, we observed significant increases in the levels of HIF-1 $\beta$, TIGAR, GLUT1, and GLUT3 in the ipsilateral side of the brain after UCCAO. These observations are consistent with the previous reports and help confirm the success of achieving $\mathrm{CCH}$ in the UCCAO mice.

In the present study by using the well-established UCCAO on 4.5-6 month old mice, we demonstrated that $\mathrm{CCH}$ caused anxiety, motor function deficits, and mild impairment of cognitive functions. These findings provide experimental evidence that $\mathrm{CCH}$ alone can lead to memory deficits. $\mathrm{CCH}$ for more than one month resulted in only mild cognitive deficits, indicating a large reserved capacity of the brain. Pimentel-Coelho et al. (2013) performed UCCAO on 4-month-old mice and did not find any cognitive deficits tested by using T-water maze task five weeks after UCCAO, but the same approach led to spatial learning impairments in APPswe/PS1 mice (Pimentel-Coelho et al., 2013). Our observations that mild cognitive impairment of the $\mathrm{CCH}$ mice was detectable only in the more sensitive reversal MWM, but not in the original MWM, are consistent with the above report. CCH's initiation of cognitive impairment may be aggravated by other insults (such as overexpression of APPswe/PS1). A synergistic effects between overexpression of mutated APP and $\mathrm{CCH}$ on learning impairment in a mouse model, which over-expresses human APP bearing both the Swedish and Indiana mutations (APPSw/Ind-Tg mice), has been observed previously (Yamada et al., 2011). It is interesting for future studies to investigate the impact of $\mathrm{CCH}$ for a much longer time on cognitive function since many individuals could have cerebrovascular deficits years before the onset of memory loss in $\mathrm{AD}$.

To investigate the possible molecular mechanisms that might underlie the $\mathrm{CCH}$-induced cognitive impairment, we further investigated several AD-related molecules and pathways in the $\mathrm{CCH}$ mouse brain. Because O-GlcNAcylation is a sensor of intracellular glucose metabolism (Zachara and Hart, 2004) and is expected to be decreased under the hypoperfusion condition, we investigated protein O-GlcNAcylation. We found a significant decrease in the O-GlcNAcylation level in both the cortex and the hippocampus of the ipsilateral hemisphere of the UCCAO. These results indicate that the compensative increase in GLUT1 and GLUT3 might fail to compensate the intracellular glucose to the normal level. The reduction of O-GlcNAcylation level may result from a reduction of the intracellular level of UDP-GlcNAc, which is the donor for protein O-GlcNAcylation and is also the major activator of OGT activity, because UDP-GlcNAc is synthesized from glucose metabolism HBP. As we also found a reduction of OGT level in the hippocampus of the $\mathrm{CCH}$ brain, this reduction might also contribute to the decrease of O-GlcNAcylation in the hippocampus of the $\mathrm{CCH}$ mice.
It has been demonstrated that abnormal hyperphosphorylation of tau is crucial to neurodegeneration in $\mathrm{AD}$ and probably also in other tauopathies (Gong and Iqbal, 2008; Iqbal etal., 2013). Tau hyperphosphorylation can be promoted by several factors. One of these factors is decreased brain glucose metabolism through down-regulation of tau O-GlcNAcylation because the latter regulates tau phosphorylation inversely (Liu et al., 2004, 2009; Li et al., 2006). We therefore studied site-specific tau phosphorylation and found elevated tau phosphorylation at two sites (Thr212 and Ser262) in the cerebral cortex and six sites (Ser199, Thr205, Thr212, Ser214, Thr231, and Ser262) among the eight phosphorylation sites studied in the hippocampus in mice after $\mathrm{CCH}$. Elevated tau phosphorylation at more sites in the hippocampus than in the cortex suggests that the hippocampus is more vulnerable to $\mathrm{CCH}$-induced tau hyperphosphorylation. Higher vulnerability of the hippocampus to fasting-induced reduction of O-GlcNAcylation and elevation of tau phosphorylation than the cerebral cortex were also seen previously (Li et al., 2006). This differentiative vulnerability of tau hyperphosphorylation is fully consistent to the fact that the hippocampus is more vulnerable to neurodegeneration than the cerebral cortex in $\mathrm{AD}$, further supporting the role of $\mathrm{CCH}$ in the development of $\mathrm{AD}$. Taken together, these findings suggest that $\mathrm{CCH}$ leads to decreased O-GlcNAcylation and then hyperphosphorylation of tau. Abnormally hyperphosphorylated tau might also contribute to the increased anxiety observed in the $\mathrm{CCH}$ mice since a link between anxiety-related behavior and tau hyperphosphorylation was reported previously (Pristera et al., 2013).

It is surprising that the increased tau phosphorylation was also seen at the contralateral side, where O-GlcNAcylation was not decreased, in the UCCAO mice. These observations suggest that an additional mechanism might be involved in tau hyperphosphorylation at the contralateral side of the UCCAO mice. Alternatively, it is possible that the mice had produced sufficient cross circulation between two hemispheres during the 2.5 month period after UCCAO leading to a significant reduction of the perfusion in the contralateral hemisphere due to blood steal, although this phenomenon was not seen 28 days after UCCAO (Kitagawa et al., 2005). This possibility is supported by our observations of increased GLUT1 level in the contralateral cortex and a clear tendency of increased GLUT3 level in the contralateral cortex and hippocampus. Alterations of synaptic proteins and some insulin signaling proteins, as well as slight increase in the number of degenerative neurons, were also seen in the contralateral side of the mouse brains after UCCAO.

Synapses are the structural basis of neural connection, neural plasticity and cognitive function. The clinical symptoms of AD correlate highly to the synaptic loss in the brain (Scheff et al., 2011). Elevated levels of synaptic proteins, such as of synaptophysin, syntaxin, and SNAP-25, are observed in human brains with Braak stages 3 and 4 (Mukaetova-Ladinska et al., 2000), whereas loss of synaptic proteins is a relatively late phenomenon in $\mathrm{AD}$ brain, occurring well after the onset of clinically detectable dementia and the appearance of $\mathrm{A} \beta$ plaques and NTFs (Mukaetova-Ladinska et al., 2000). The present study also showed altered levels of synaptic proteins, including increased pre-synaptic protein synapsin and 
post-synaptic protein PSD95, as well as decreased pre-synaptic protein synaptophysin, in the cerebral cortex and/or the hippocampus of the $\mathrm{CCH}$ mice 2.5 months after UCCAO. These alterations of the synaptic proteins suggest alterations of synaptic vesicle integrity and functions and might underlie the molecular basis of the mild cognitive deficits of the $\mathrm{CCH}$ mice. Decreased level of synaptophysin and alterations of synaptic ultrastructure in the CA1 area of the hippocampus have been observed in rat brains after chronic cerebrovascular hypoperfusion (Wang et al., 2010).

Recent studies have established that brain insulin signaling plays an important role in the regulation of neuronal activity and neural plasticity (van der Heide etal., 2006; Reagan, 2007). Dysregulation of brain insulin signaling has been reported in AD (Frolich etal., 1999; Rivera etal., 2005; Steen et al., 2005; Liu etal., 2011). Various changes of the insulin signaling pathway are seen in different models of neurodegenerative disorders (Pedersen and Flynn, 2004; Chen et al., 2012, 2013). The different changes of insulin signaling in $\mathrm{AD}$ and in these models might represent a diversity of insults, which might ultimately contribute to cognitive impairment. In the present study, majority of the alterations of the insulin signaling pathway was increases rather than decreases, which might represent possible compensation in response to $\mathrm{CCH}$. These compensative changes resulted in a marked increase in the inhibitory Ser9 phosphorylation of GSK-3 $\beta$ and thus a marked inhibition of its kinase activity. However, the GSK-3 $\beta$ phosphorylation sites of tau were hyperphosphorylated rather than hypophosphorylated in the $\mathrm{CCH}$ mouse brains, suggesting that other opposing factors, such as reduction of O-GlcNAcylation and probably activation of some other tau kinases, had counteracted the effect of GSK-3 $\beta$ inhibition on tau phosphorylation after $\mathrm{CCH}$.

No detectable ischemia or cell death were observed previously in the brains of 3-4 months old mice after UCCAO (Kitagawa et al., 2005; Pimentel-Coelho et al., 2013). In the present study, however, we found degenerative neurons by using Fluoro-Jade staining in the brains, especially in the granule neurons of the dentate gyrus, of the brains in 7-8.5 months old CCH mice. Furthermore, these degenerative neurons were filled with hyperphosphorylated tau, suggesting that neurodegeneration might be attributed to tau phosphorylation in the $\mathrm{CCH}$ mouse brains. As the granule neurons of the dentate gyrus receive inputs from the entorhinal cortex through the perforant pathway and send signals to the CA3 pyramidal neurons through the mossy fiber pathway, which makes the critical pathways for memory processing and recording, neuronal degeneration in these areas, together with the synaptic deficits, might underlie the basis of mild memory impairments observed in the $\mathrm{CCH}$ mice in the present study.

In conclusion, the present study shows that $\mathrm{CCH}$ induced by UCCAO decreased the protein O-GlcNAcylation, increased tau phosphorylation, dysregulated synaptic proteins and brain insulin signaling, and caused mild cognitive impairment. These studies provide experimental evidence showing the possible mechanisms by which $\mathrm{CCH}$ can contribute to cognitive impairment and $\mathrm{AD}$ probably through reduction of protein
O-GlcNAcylation, hyperphosphorylation of tau, dysregulated synaptic plasticity and neural degeneration in the brain. These findings also provide a possible mechanistic linkage between AD and $\mathrm{VaD}$.

\section{AUTHOR CONTRIBUTIONS}

Conception of the research: Cheng-Xin Gong, Yang Zhao, Jin-hua $\mathrm{Gu}$, Qun Liu, Khalid Iqbal, and Fei Liu. Performing experiments: Yang Zhao, Jin-hua Gu, and Chun-ling Dai. Analyses and interpretation of results: Yang Zhao, Jin-hua Gu, Chun-ling Dai, Qun Liu, Khalid Iqbal, Fei Liu, and Cheng-Xin Gong. Drafting of the manuscript: Yang Zhao. Critical revision of the manuscript: Cheng-Xin Gong, Yang Zhao, Jin-hua Gu, Cheng-Xin Dai, Qun Liu, Khalid Iqbal, and Fei Liu.

\section{ACKNOWLEDGMENTS}

This work was supported in part by the First Hospital of Jilin University, China, and the New York State Office for People with Developmental Disabilities as well as grants from the National Natural Science Foundation of China (81000496) and the U.S. Alzheimer's Association (IIRG-10-170405). The funders had no role in study design, data collection and analysis, decision to publish, or preparation of the manuscript.

\section{REFERENCES}

Adibhatla, R. M., and Hatcher, J. F. (2008). Phospholipase A(2), reactive oxygen species, and lipid peroxidation in CNS pathologies. BMB Rep. 41, 560-567. doi: 10.5483/BMBRep.2008.41.8.560

Bang, J., Jeon, W. K., Lee, I. S., Han, J. S., and Kim, B. Y. (2013). Biphasic functional regulation in hippocampus of rat with chronic cerebral hypoperfusion induced by permanent occlusion of bilateral common carotid artery. PLoS ONE 8:e70093. doi: 10.1371/journal.pone.0070093

Battistin, L., and Cagnin, A. (2010). Vascular cognitive disorder. A biological and clinical overview. Neurochem. Res. 35, 1933-1938. doi: 10.1007/s11064-0100346-5

Bensaad, K., Tsuruta, A., Selak, M. A., Vidal, M. N., Nakano, K., Bartrons, R., et al. (2006). TIGAR, a p53-inducible regulator of glycolysis and apoptosis. Cell 126, 107-120. doi: 10.1016/j.cell.2006.05.036

Bevins, R. A., and Besheer, J. (2006). Object recognition in rats and mice: a onetrial non-matching-to-sample learning task to study 'recognition memory'. Nat. Protoc. 1, 1306-1311. doi: 10.1038/nprot.2006.205

Cardoso, S., Correia, S., Santos, R. X., Carvalho, C., Santos, M. S., Oliveira, C. R., et al. (2009). Insulin is a two-edged knife on the brain. J. Alzheimers Dis. 18, 483-507. doi: 10.3233/JAD-2009-1155

Carter, R. J., Lione, L. A., Humby, T., Mangiarini, L., Mahal, A., Bates, G. P., et al. (1999). Characterization of progressive motor deficits in mice transgenic for the human Huntington's disease mutation. J. Neurosci. 19, 3248-3257.

Chan, J. Y., Tsai, C. Y., Wu, C. H., Li, F. C., Dai, K. Y., Sun, E. Y., et al. (2011). Sumoylation of hypoxia-inducible factor-1 alpha ameliorates failure of brain stem cardiovascular regulation in experimental brain death. PLoS ONE 6:e17375. doi: 10.1371/journal.pone.0017375

Chen, Y., Liang, Z., Tian, Z., Blanchard, J., Dai, C. L., Chalbot, S., et al. (2013). Intracerebroventricular streptozotocin exacerbates Alzheimer-like changes of 3xTg-AD mice. Mol. Neurobiol. doi: 10.1007/s12035-013-8539-y

Chen, Y., Tian, Z., Liang, Z., Sun, S., Dai, C. L., Lee, M. H., et al. (2012). Brain gene expression of a sporadic (icv-STZ Mouse) and a familial mouse model (3xTg-AD mouse) of Alzheimer's disease. PLoS ONE 7:e51432. doi: 10.1371/journal.pone.0051432

Crawford, G. L., Hart, G. W., and Whiteheart, S. W. (2008). Murine platelets are not regulated by $\mathrm{O}-$ linked beta-N-acetylglucosamine. Arch. Biochem. Biophys. 474, 220-224. doi: 10.1016/j.abb.2008.03.014

D'Hooge, R., and De Deyn, P. P. (2001). Applications of the Morris water maze in the study of learning and memory. Brain Res. Brain Res. Rev. 36, 60-90. doi: S0165017301000674 
Dubois, M. F., and Hébert, R. (2001). The incidence of vascular dementia in Canada: a comparison with Europe and East Asia. Neuroepidemiology 20, 179-187. doi: $10.1159 / 000054785$

Ennaceur, A., and Delacour, J. (1988). A new one-trial test for neurobiological studies of memory in rats. 1: behavioral data. Behav. Brain Res. 31, 47-59. doi: 0166-4328(88)90157-X

Erecińska, M., and Silver, I. A. (1989). ATP and brain function. J. Cereb. Blood Flow Metab. 9, 2-19. doi: 10.1038/jcbfm.1989.2

Farkas, E., Luiten, P. G., and Bari, F. (2007). Permanent, bilateral common carotid artery occlusion in the rat: a model for chronic cerebral hypoperfusion-related neurodegenerative diseases. Brain Res. Rev. 54, 162-180. doi: 10.1016/j.brainresrev.2007.01.003

Frolich, L., Blum-Degen, D., Riederer, P., and Hoyer, S. (1999). A disturbance in the neuronal insulin receptor signal transduction in sporadic Alzheimer's disease. Ann. N. Y. Acad. Sci. 893, 290-293. doi: 10.1111/j.1749-6632.1999.tb07839.x

Gerozissis, K. (2008). Brain insulin, energy and glucose homeostasis; genes, environment and metabolic pathologies. Eur. J. Pharmacol. 585, 38-49. doi 10.1016/j.ejphar.2008.01.050

Gong, C. X., and Iqbal, K. (2008). Hyperphosphorylation of microtubule-associated protein tau: a promising therapeutic target for Alzheimer disease. Curr. Med. Chem. 15, 2321-2328. doi: 10.2174/092986708785909111

Grundke-Iqbal, I., Iqbal, K., Quinlan, M., Tung, Y. C., Zaidi, M. S., and Wisniewski, H. M. (1986a). Microtubule-associated protein tau. A component of Alzheimer paired helical filaments. J. Biol. Chem. 261, 6084-6089.

Grundke-Iqbal, I., Iqbal, K., Tung, Y. C., Quinlan, M., Wisniewski, H. M., and Binder, L. I. (1986b). Abnormal phosphorylation of the microtubule-associated protein tau (tau) in Alzheimer cytoskeletal pathology. Proc. Natl. Acad. Sci. U.S.A. 83, 4913-4917. doi: 10.1073/pnas.83.13.4913

Hanover, J. A., Krause, M. W., and Love, D. C. (2010). The hexosamine signaling pathway: O-GlcNAc cycling in feast or famine. Biochim. Biophys. Acta 1800, 80-95. doi: 10.1016/j.bbagen.2009.07.017

Hoshino, A., Matoba, S., Iwai-Kanai, E., Nakamura, H., Kimata, M., Nakaoka, M., et al. (2012). p53-TIGAR axis attenuates mitophagy to exacerbate cardiac damage after ischemia. J. Mol. Cell. Cardiol. 52, 175-184. doi: 10.1016/j.yjmcc.2011.10.008

Iqbal, K., Gong, C. X., and Liu, F. (2013). Hyperphosphorylation-induced tau oligomers. Front. Neurol. 4:112. doi: 10.3389/fneur.2013.00112

Iwabuchi, S., and Kawahara, K. (2011). Inducible astrocytic glucose transporter-3 contributes to the enhanced storage of intracellular glycogen during reperfusion after ischemia. Neurochem. Int. 59, 319-325. doi: 10.1016/j.neuint.2011.06.006

Kalaria, R. N., Akinyemi, R., and Ihara, M. (2012). Does vascular pathology contribute to Alzheimer changes? J. Neurol. Sci. 322, 141-147. doi: 10.1016/j.jns.2012.07.032

Kammoun, S., Gold, G., Bouras, C., Giannakopoulos, P., McGee, W., Herrmann, F., et al. (2000). Immediate causes of death of demented and non-demented elderly. Acta Neurol. Scand. Suppl. 176, 96-99. doi: 10.1034/j.1600-0404.2000.00314.x

Kimata, M., Matoba, S., Iwai-Kanai, E., Nakamura, H., Hoshino, A., Nakaoka, M., et al. (2010). p53 and TIGAR regulate cardiac myocyte energy homeostasis under hypoxic stress. Am. J. Physiol. Heart Circ. Physiol. 299, H1908-H1916. doi: 10.1152/ajpheart.00250.2010

Kitagawa, K., Yagita, Y., Sasaki, T., Sugiura, S., Omura-Matsuoka, E., Mabuchi, T., et al. (2005). Chronic mild reduction of cerebral perfusion pressure induces ischemic tolerance in focal cerebral ischemia. Stroke 36, 2270-2274. doi: 01.STR.0000181075.77897.0e

Lee, J. S., Im, D. S., An, Y. S., Hong, J. M., Gwag, B. J., and Joo, I. S. (2011). Chronic cerebral hypoperfusion in a mouse model of Alzheimer's disease: an additional contributing factor of cognitive impairment. Neurosci. Lett. 489, 84-88. doi: 10.1016/j.neulet.2010.11.071

Leszek, J., Sochocka, M., and Gasiorowski, K. (2012). Vascular factors and epigenetic modifications in the pathogenesis of Alzheimer's disease. J. Neurol. Sci. 323, 25-32. doi: 10.1016/j.jns.2012.09.010

Levine, S. (1960). Anoxic-ischemic encephalopathy in rats. Am. J. Pathol. 36, 1-17. doi: 10.1016/0014-4886(66)90082-3

Li, X., Lu, F., Wang, J. Z., and Gong, C. X. (2006). Concurrent alterations of O-GlcNAcylation and phosphorylation of tau in mouse brains during fasting. Eur. J. Neurosci. 23, 2078-2086. doi: 10.1111/j.1460-9568.2006. 04735. $\mathrm{x}$

Liu, F., Iqbal, K., Grundke-Iqbal, I., Hart, G. W., and Gong, C. X. (2004). O-GlcNAcylation regulates phosphorylation of tau: a mechanism involved in
Alzheimer's disease. Proc. Natl. Acad. Sci. U.S.A. 101, 10804-10809. doi: 10.1073/pnas.0400348101

Liu, F., Shi, J., Tanimukai, H., Gu, J., Grundke-Iqbal, I., Iqbal, K., et al. (2009). Reduced O-GlcNAcylation links lower brain glucose metabolism and tau pathology in Alzheimer's disease. Brain 132, 1820-1832. doi: 10.1093/brain/ awp099

Liu, F., Zaidi, T., Iqbal, K., Grundke-Iqbal, I., and Gong, C. X. (2002). Aberrant glycosylation modulates phosphorylation of tau by protein kinase A and dephosphorylation of tau by protein phosphatase 2A and 5. Neuroscience 115, 829-837. doi: S0306452202005109

Liu, Y., Liu, F., Grundke-Iqbal, I., Iqbal, K., and Gong, C. X. (2011). Deficient brain insulin signalling pathway in Alzheimer's disease and diabetes. J. Pathol. 225, 54-62. doi: 10.1002/path.2912

Meyer, J. S., Rauch, G., Rauch, R. A., and Haque, A. (2000). Risk factors for cerebral hypoperfusion, mild cognitive impairment, and dementia. Neurobiol. Aging 21, 161-169. doi: S0197-4580(00)00136-6

Mukaetova-Ladinska, E. B., Garcia-Siera, F., Hurt, J., Gertz, H. J., Xuereb, J. H., Hills, R., et al. (2000). Staging of cytoskeletal and beta-amyloid changes in human isocortex reveals biphasic synaptic protein response during progression of Alzheimer's disease. Am. J. Pathol. 157, 623-636. doi: S0002-9440(10) 64573-7

Pedersen, W. A., and Flynn, E. R. (2004). Insulin resistance contributes to aberrant stress responses in the Tg2576 mouse model of Alzheimer's disease. Neurobiol. Dis. 17, 500-506. doi: 10.1016/j.nbd.2004. 08.003

Peters, A. (2011). The selfish brain: Competition for energy resources. Am. J. Hum. Biol. 23, 29-34. doi: 10.1002/ajhb.21106

Pimentel-Coelho, P. M., Michaud, J. P., and Rivest, S. (2013). Effects of mild chronic cerebral hypoperfusion and early amyloid pathology on spatial learning and the cellular innate immune response in mice. Neurobiol. Aging 34, 679-693. doi: 10.1016/j.neurobiolaging.2012.06.025

Pristera, A., Saraulli, D., Farioli-Vecchioli, S., Strimpakos, G., Costanzi, M., di Certo, M. G., etal. (2013). Impact of N-tau on adult hippocampal neurogenesis, anxiety, and memory. Neurobiol. Aging 34, 2551-2563. doi: 10.1016/j.neurobiolaging.2013.05.010

Qutub, A. A., and Hunt, C. A. (2005). Glucose transport to the brain: a systems model. Brain Res. Brain Res. Rev. 49, 595-617. doi: 10.1016/j.brainresrev.2005.03.002

Reagan, L. P. (2007). Insulin signaling effects on memory and mood. Curr. Opin. Pharmacol. 7, 633-637. doi: 10.1016/j.coph.2007.10.012

Rice, J. E. III, Vannucci, R. C., and Brierley, J. B. (1981). The influence of immaturity on hypoxic-ischemic brain damage in the rat. Ann. Neurol. 9, 131-141. doi: 10.1002/ana.410090206

Rivera, E. J., Goldin, A., Fulmer, N., Tavares, R., Wands, J. R., and de la Monte, S. M. (2005). Insulin and insulin-like growth factor expression and function deteriorate with progression of Alzheimer's disease: link to brain reductions in acetylcholine. J. Alzheimers Dis. 8, 247-268.

Roman, G. C. (2002). Vascular dementia revisited: diagnosis, pathogenesis, treatment, and prevention. Med. Clin. North Am. 86, 477-499. doi: 10.1016/S00257125(02)00008-1

Sarti, C., Pantoni, L., Bartolini, L., and Inzitari, D. (2002). Persistent impairment of gait performances and working memory after bilateral common carotid artery occlusion in the adult Wistar rat. Behav. Brain Res. 136, 13-20. doi: S0166432802000906

Sato, N., and Morishita, R. (2013). Roles of vascular and metabolic components in cognitive dysfunction of Alzheimer disease: short- and long-term modification by non-genetic risk factors. Front. Aging Neurosci. 5:64. doi: 10.3389/fnagi.2013.00064

Scheff, S. W., Price, D. A., Schmitt, F. A., Scheff, M. A., and Mufson, E. J. (2011). Synaptic loss in the inferior temporal gyrus in mild cognitive impairment and Alzheimer's disease. J. Alzheimers Dis. 24, 547-557. doi: 10.3233/JAD-2011-101782

Singh, N., Sharma, G., and Mishra, V. (2012). Hypoxia inducible factor-1: its potential role in cerebral ischemia. Cell. Mol. Neurobiol. 32, 491-507. doi: 10.1007/s10571-012-9803-9

Slattery, D. A., and Cryan, J. F. (2012). Using the rat forced swim test to assess antidepressant-like activity in rodents. Nat. Protoc. 7, 1009-1014. doi: 10.1038/nprot.2012.044 
Steen, E., Terry, B. M., Rivera, E. J., Cannon, J. L., Neely, T. R., Tavares, R., et al. (2005). Impaired insulin and insulin-like growth factor expression and signaling mechanisms in Alzheimer's disease-is this type 3 diabetes? J. Alzheimers Dis. 7, 63-80.

Sudduth, T. L., Powell, D. K., Smith, C. D., Greenstein, A., and Wilcock, D. M. (2013). Induction of hyperhomocysteinemia models vascular dementia by induction of cerebral microhemorrhages and neuroinflammation. J. Cereb. Blood Flow Metab. 33, 708-715. doi: 10.1038/jcbfm.2013.1

Tatebayashi, Y., Iqbal, K., and Grundke-Iqbal, I. (1999). Dynamic regulation of expression and phosphorylation of tau by fibroblast growth factor-2 in neural progenitor cells from adult rat hippocampus. J. Neurosci. 19, 5245-5254.

Urabe, T. (2012). [Molecular mechanism and new protective strategy for ischemic white matter damages]. Rinsho Shinkeigaku 52, 908-910. doi: DN/JST.JSTAGE/clinicalneurol/52.908

Valerio Romanini, C., Dias Fiuza Ferreira, E., Correia Bacarin, C., Verussa, M. H., Weffort de Oliveira, R. M., and Milani, H. (2013). Neurohistological and behavioral changes following the four-vessel occlusion/internal carotid artery model of chronic cerebral hypoperfusion: comparison between normotensive and spontaneously hypertensive rats. Behav. Brain Res. 252, 214-221. doi: 10.1016/j.bbr.2013.05.043

van der Heide, L. P., Ramakers, G. M., and Smidt, M. P. (2006). Insulin signaling in the central nervous system: learning to survive. Prog. Neurobiol. 79, 205-221. doi: 10.1016/j.pneurobio.2006.06.003

Vannucci, S. J., Maher, F., and Simpson, I. A. (1997). Glucose transporter proteins in brain: delivery of glucose to neurons and glia. Glia 21, 2-21. doi: 10.1002/(SICI) 1098-1136(199709)21:1 < 2::AID-GLIA2>3.0.CO;2-C

Vannucci, S. J., Reinhart, R., Maher, F., Bondy, C. A., Lee, W. H., Vannucci, R. C., et al. (1998). Alterations in GLUT1 and GLUT3 glucose transporter gene expression following unilateral hypoxia-ischemia in the immature rat brain. Brain Res. Dev. Brain Res. 107, 255-264. doi: S0165380698000212

Walf, A. A., and Frye, C. A. (2007). The use of the elevated plus maze as an assay of anxiety-related behavior in rodents. Nat. Protoc. 2, 322-328. doi: 10.1038/nprot.2007.44

Wang, F., Wang, Y., Geng, X., Asmaro, K., Peng, C., Sullivan, J. M., et al. (2012a) Neuroprotective effect of acute ethanol administration in a rat with transien cerebral ischemia. Stroke. 43, 205-210. doi: 10.1161/STROKEAHA.111.629576

Wang, Z., Tsai, L. K., Munasinghe, J., Leng, Y., Fessler, E. B., Chibane, F., et al. (2012b). Chronic valproate treatment enhances postischemic angiogenesis and promotes functional recovery in a rat model of ischemic stroke. Stroke 43, 2430-2436. doi: 10.1161/STROKEAHA.112.652545

Wang, X., Xing, A., Xu, C., Cai, Q., Liu, H., and Li, L. (2010). Cerebrovascular hypoperfusion induces spatial memory impairment, synaptic changes, and amyloid-beta oligomerization in rats. J. Alzheimers Dis. 21, 813-822. doi: 10.3233/JAD-2010-100216
Watanabe, M., Masaoka, N., Nakajima, Y., Nagaishi, M., and Yamamoto, T. (2009). Changes of expression of glucose transporters in the fetal lamb brain after MCI-186 administration to the maternal circulation with 10 -min persistent umbilical cord occlusion. J. Matern. Fetal Neonatal Med. 22, 829-836. doi: 10.1080/14767050902801702

Yamada, M., Ihara, M., Okamoto, Y., Maki, T., Washida, K., Kitamura, A., et al. (2011). The influence of chronic cerebral hypoperfusion on cognitive function and amyloid beta metabolism in APP overexpressing mice. PLoS ONE 6:e16567. doi: 10.1371/journal.pone.0016567

Yan, J., Zhou, B., Taheri, S., and Shi, H. (2011). Differential effects of HIF-1 inhibition by $\mathrm{YC}-1$ on the overall outcome and blood-brain barrier damage in a rat model of ischemic stroke. PLoS ONE 6:e27798. doi: 10.1371/journal.pone.0027798

Yu, S., Zhao, T., Guo, M., Fang, H., Ma, J., Ding, A., et al. (2008). Hypoxic preconditioning up-regulates glucose transport activity and glucose transporter (GLUT1 and GLUT3) gene expression after acute anoxic exposure in the cultured rat hippocampal neurons and astrocytes. Brain Res. 1211, 22-29. doi: 10.1016/j.brainres.2005.04.029

Yuan, L. B., Dong, H. L., Zhang, H. P., Zhao, R. N., Gong, G., Chen, X. M., et al. (2011). Neuroprotective effect of orexin-A is mediated by an increase of hypoxia-inducible factor-1 activity in rat. Anesthesiology 114, 340-354. doi: 10.1097/ALN.0b013e318206ff6f

Zachara, N. E., and Hart, G. W. (2004). O-GlcNAc a sensor of cellular state: the role of nucleocytoplasmic glycosylation in modulating cellular function in response to nutrition and stress. Biochim. Biophys. Acta 1673, 13-28. doi: 10.1016/j.bbagen.2004.03.016

Conflict of Interest Statement: The authors declare that the research was conducted in the absence of any commercial or financial relationships that could be construed as a potential conflict of interest.

Received: 04 January 2014; paper pending published: 16 January 2014; accepted: 16 January 2014; published online: 10 February 2014.

Citation: Zhao Y, Gu J-h, Dai C-l, Liu Q, Iqbal K, Liu F and Gong C-X (2014) Chronic cerebral hypoperfusion causes decrease of O-GlcNAcylation, hyperphosphorylation of tau and behavioral deficits in mice. Front. Aging Neurosci. 6:10. doi: 10.3389/fnagi.2014.00010

This article was submitted to the journal Frontiers in Aging Neuroscience.

Copyright (C) 2014 Zhao, Gu, Dai, Liu, Iqbal, Liu and Gong. This is an open-access article distributed under the terms of the Creative Commons Attribution License (CC BY). The use, distribution or reproduction in other forums is permitted, provided the original author(s) or licensor are credited and that the original publication in this journal is cited, in accordance with accepted academic practice. No use, distribution or reproduction is permitted which does not comply with these terms. 